\title{
EXISTENCE OF HETEROCLINIC ORBITS FOR SYSTEMS SATISFYING MONOTONICITY CONDITIONS
}

\author{
BOGDAN KAŹMIERCZAK ${ }^{1,2}$, VITALY VOLPERT ${ }^{3}$ \\ ${ }^{1}$ Insitute of Fundamental Technological Research \\ Świetokrzyska 21, 00-049 Warsaw, Poland, email: bkazmier@ippt.gov.pl \\ 2 Department of Mathematics and the Interdisciplinary Center for the Study of \\ Biocomplexity, University of Notre Dame, Notre Dame, IN 46556-5670 \\ ${ }^{3}$ Laboratoire de Mathématiques Appliquées, UMR 5585 CNRS \\ Université Lyon 1, 69622 Villeurbanne, France, email: volpert@maply.univ-lyon1.fr
}

\begin{abstract}
We prove existence of heteroclinic orbits for systems of ordinary differential equations satisfying monotonicity conditions. The proof is carried out by means of the implicit function theorem. We apply these results to prove existence of travelling waves for the system describing a multicomponent plasma sustained by a laser beam.
\end{abstract}

Key words and phrases: reaction-diffusion systems, implicit function theorem, heteroclinic orbits.

\section{Introduction}

We study the existence of travelling waves solutions of the parabolic system of equations

$$
\frac{\partial F_{i}\left(T_{i}\right)}{\partial t}=\frac{\partial}{\partial x}\left(a_{i}\left(T_{i}\right) \frac{\partial T_{i}}{\partial x}\right)+f_{i}(T), \quad i=1, \ldots, n
$$

arising in various applications. Here $T=\left(T_{1}, \ldots, T_{n}\right)$. A travelling wave solution is a solution of the form $T(x, t)=u(x-q t)$, where $q$, the wave velocity is an unknown constant, and the function $u(x)$ satisfies the system of equations

$$
\left(a_{i}\left(u_{i}\right) u_{i}^{\prime}\right)^{\prime}-q\left(F_{i}\left(u_{i}\right)\right)^{\prime}+f_{i}(u)=0, \quad i=1, \ldots, n,
$$

where prime denotes the derivative with respect to $\xi:=x-q t$. Taking into account other possible applications, we will study the following more general system of equations

$$
a_{i}\left(u_{i}, u_{i}^{\prime}\right) u_{i}^{\prime \prime}-q c_{i}\left(u_{i}, u_{i}^{\prime}\right) u_{i}^{\prime}+M_{i}\left(u, u_{i}^{\prime}\right) u_{i}^{\prime}+f_{i}(u)=0, \quad i=1, \ldots, n .
$$


We assume that $f\left(u_{+}\right)=f\left(u_{-}\right)=0$ for some constant vectors $u_{+}$and $u_{-}$and we look for solutions having limits at infinity,

$$
\lim _{\xi \rightarrow \pm \infty} u(\xi)=u_{ \pm} \cdot
$$

The constant $q$ in (1.3) should be found together with the function $u(\xi)$ satisfying (1.3), (1.4).

We consider the class of systems satisfying the following conditions:

$$
\frac{\partial f_{i}}{\partial u_{j}} \geq 0, \quad \frac{\partial M_{i}}{\partial u_{j}} \geq 0, \quad i \neq j .
$$

These are so-called monotone systems for which comparison theorems are applicable. Existence of travelling waves for monotone systems was studied for various particular cases of the system (1.3) (see [20], [21] and references therein). The authors of [15] considered the case where $a_{i} \equiv c_{i} \equiv$ const, $M_{i} \equiv 0$, and in [4] $a_{i}$ and $c_{i}$ were constant but $M_{i}$ was a function of $u$ and $u_{i}^{\prime}$.

There are different methods to study existence of waves for monotone systems. In [15] the Leray-Schauder method is used. It is based on the application of the topological degree defined for elliptic operators in unbounded domains [17], [18], [19]. In [11], [1] or [8] the Conley index theory is applied. In this work we use the implicit function theorem and the continuation method. We consider an appropriate system depending on a real parameter $\lambda \in[0,1]$ in such a way that for $\lambda=1$ it coincides with system (1.3) and for $\lambda=0$ it becomes a system, for which the considered heteroclinic orbits exist. Here we use the results of [15]. First, we prove that for all the possible strictly monotone heteroclinic solutions both $\left|u^{\prime}\right|_{C^{1}}$ and $|q|$ are bounded from above by constants independent of $\lambda \in[0,1]$. Starting from the unique strictly monotone heteroclinic solution for $\lambda=0$, by means of the implicit function theorem, we can show that the unique heteroclinic solution exists also for all $\lambda>0$ sufficiently small. Having shown that heteroclinic solutions $u_{\lambda}$ are strictly monotone for $\lambda \in\left[0, \lambda_{1}\right], \lambda_{1}$ sufficiently close to 0 , we can repeat the procedure. The monotonicity property enables us to take advantage of a priori estimates and allows us to demonstrate that the linearization of the mapping generated by the left-hand sides of the equations is boundedly invertible. It allows us to extend the interval of existence of heteroclinic pairs. It is necessary to emphasize that in this procedure the monotonicity conditions are crucial. As the heteroclinic pair of the starting system $(\lambda=0)$ is unique, then we obtain the uniqueness of solutions at every stage of continuation.

The contents of the paper are as follows. In Section 2 we specify the assumptions on the system. In the following section we study properties of linearized operators and in Section 4 we prove existence of waves. In Section 5 we consider possible generalizations of the results and in Section 6 we consider a particular case where the proof is self contained. In Section 7 we show that this method can be applied to prove the existence of travelling wave solutions in a multitemperature model of laser sustained plasma. These waves connect two states of the gas: the cold unionized and a hot ionized one. In the last section we consider systems of ODEs perturbed by terms with delays. If these terms are sufficiently small, the heteroclinic solutions persist after the perturbation, if they exist for the unperturbed system. 


\section{Basic assumptions}

We consider the problem (1.3), (1.4) assuming that conditions (1.5) are satisfied. To simplify the presentation we suppose that the first inequality in (1.5) is strict (see [16]).

We study heteroclinic solutions, which are strictly monotone functions of $\xi$, i.e. such that $u_{i}^{\prime}(\xi)>0$ for all $\xi \in \mathbb{R}^{1}$. Thus $u_{+}>u_{-}$, where the inequality between the vectors is understood componentwise. Without loss of generality we can assume that $u_{+}=\mathbf{0}, u_{-}=\mathbf{1}$. Here $\mathbf{0}$ and $\mathbf{1}$ are vectors with all their components equal 0 and 1 , respectively.

We consider in this work the bistable case where all eigenvalues of the matrices $\operatorname{Df}(\mathbf{0})$ and $D f(\mathbf{1})$ are in the left-half plane. We assume that the vector-function $f(u)$ has a finite number of zeros $E^{j}=\left(e_{1}^{j}, \ldots, e_{n}^{j}\right) \in(0,1)^{n}, j=1, \ldots, K$. Each matrix $D f\left(E^{j}\right)$ has at least one eigenvalue in the right-half plane.

We recall that due to the Perron-Frobenius theorem the principal eigenvalue of a matrix with positive off-diagonal elements is real and simple, and the corresponding eigenvector is positive. We will use below a generalization of this theorem for linear elliptic problems satisfying the monotonicity condition [16], [20] (see Lemma 4).

The functions $a_{i}\left(u_{i}, p\right), c_{i}\left(u_{i}, p\right), M_{i}(u, p)$, and $f(u)$ are supposed to be continuous together with their second derivatives. We do not assume that they are bounded as functions of $p$. We assume that

$$
a_{i}\left(u_{i}, p\right) \geq a_{0}>0, \quad c_{i}\left(u_{i}, p\right) \geq c_{0}>0, \quad i=1, \ldots, n
$$

for some constants $a_{0}$ and $c_{0}$ and for all $u_{i} \in[0,1], p \geq 0$.

We impose additional assumptions used to obtain a priori estimates of solutions (see Lemmas 1 and 2, Section 3). We asume that there exists a positive constant $b$ such that

$$
\frac{c_{i}(u, p)}{c_{i}(v, r)} \leq b, \quad \forall u, v \in[0,1], \quad 0 \leq p \leq r, \quad i=1, \ldots, n .
$$

Moreover we assume that the derivatives $\partial a_{i}\left(u_{i}, p\right) / / \partial p$ are either nonnegative for all $u_{i}, p$, and $i$, or nonpositive, and that there exist continuous nonnegative functions $\beta_{i}(p)$, and $k(p)$ defined for $p \geq 0$, and a positive function $\chi_{i}(p)$, such that for any $u_{i} \in[0,1]$

$$
\int_{0}^{p} a_{i}\left(u_{i}, s\right) s d s \geq \chi_{i}(p),
$$

and

$$
\frac{p \beta_{i}(p)}{\chi_{i}(p)} \rightarrow 0, \quad p \rightarrow \infty, \quad\left|M_{i}(u, p)\right| \leq k(|u|)\left(1+\beta_{i}(p)\right) .
$$

These conditions are satisfied if $a_{i}, c_{i}, M_{i}$ are bounded as functions of $p$. 


\section{Properties of the linearized operator}

Let us consider a family of systems depending on the parameter $\lambda \in[0,1]$ :

$$
\mathcal{M}_{i}(\lambda, q, u)=0
$$

$i=1, \ldots, n$, where

$$
\mathcal{M}_{i}(\lambda, q, u)=a_{\lambda i}\left(u_{i}, u_{i}^{\prime}\right) u_{i}^{\prime \prime}-(1-\lambda) q u_{i}^{\prime}+\lambda\left[-q c_{i}\left(u_{i}, u_{i}^{\prime}\right) u_{i}^{\prime}+M_{i}\left(u, u_{i}^{\prime}\right) u_{i}^{\prime}\right]+f_{i}(u),
$$

and

$$
a_{\lambda i}\left(u_{i}, u_{i}^{\prime}\right)=\lambda a_{i}\left(u_{i}, u_{i}^{\prime}\right)+(1-\lambda)
$$

We will be interested in heteroclinic solutions $u(\xi)$ to system (1.3) joining the constant states $\mathbf{0}$ and $\mathbf{1 .}$

DEFINITION 1. A pair $\left(q_{\lambda}, u_{\lambda}\right) \in \mathbb{R}^{1} \times C^{2}\left(\mathbb{R}^{1}, \mathbb{R}^{n}\right)$ is called a heteroclinic pair for system (3.1), if $u_{\lambda}(\xi)$ satisfies system (3.1) for $q=q_{\lambda}, u_{\lambda}(\xi) \rightarrow \mathbf{0}$ as $\xi \rightarrow-\infty, u_{\lambda}(\xi) \rightarrow \mathbf{1}$ as $\xi \rightarrow \infty$ and $u_{\lambda}^{\prime}(\xi) \rightarrow \mathbf{0}$ as $\xi \rightarrow \pm \infty$. It is called strictly monotone, if $u_{\lambda}^{\prime}(\xi)>\mathbf{0}$ for all $\xi \in \mathbb{R}^{1}$.

For all the possible strictly monotone heteroclinic pairs of system (3.1) independently of $\lambda \in$ $[0,1]$ 'a priori' estimates of the $C^{1}$-norm and the absolute value of the parameter $q$ hold.

LEMMA 1. If $\left(q_{\lambda}, u_{\lambda}\right), \lambda \in[0,1]$, is a strictly monotone heteroclinic pair for system (3.1) then there exists a finite constant $m$ such that $\left|u_{\lambda}^{\prime}\right|_{C^{0}\left(\mathbb{R}^{1}\right)}<m$. This constant is independent of $\lambda, q_{\lambda}$ and $u_{\lambda}$.

PROOF. The proof of a more general lemma may be found in [8].

The next lemma states the boundedness of the parameter $q$.

LEMMA 2. If $\lambda \in[0,1]$ and $\left(q_{\lambda}, u_{\lambda}\right)$ is a strictly monotone heteroclinic pair satisfying system (3.1), then $\left|q_{\lambda}\right|<Q$, where $Q$ independent of $\lambda$ and $u_{\lambda}$.

PROOF. The proof (modulo slight modifications) is contained in [4] (Lemma 3.4) or in [8].

The boundedness of $q_{\lambda}$ allows us to estimate the exponential behaviour of monotone solutions near the singular points $\left(u_{\lambda}, u_{\lambda}^{\prime}\right)=(\mathbf{0}, \mathbf{0})$ and $(\mathbf{1}, \mathbf{0})$.

LEMMA 3. (see [16] Lemma 2.9 p. 164) There exist a number $\widetilde{\varepsilon}>0$, such that for all strictly monotone heteroclinic solutions $u_{\lambda}$ of the problem (3.1) with $\lambda \in[0,1]$ and $q \in[-Q, Q]$ the following estimates hold:

$$
\left|u_{\lambda}(\xi)\right| \leq K_{0} \widetilde{\varepsilon} \exp \left[\gamma\left(\xi-\xi_{0}\right)\right], \quad\left|u_{\lambda}^{\prime}(\xi)\right| \leq K_{0} \widetilde{\varepsilon} \exp \left[\gamma\left(\xi-\xi_{0}\right)\right],
$$


for all $\xi \leq \xi_{0}$ and $\xi_{0}$ such that $\left|u_{\lambda}\left(\xi_{0}\right)\right| \leq \widetilde{\varepsilon}$, and

$$
\left|u_{\lambda}(\xi)-1\right| \leq K_{1} \widetilde{\varepsilon} \exp \left[-\vartheta\left(\xi-\xi_{0}\right)\right], \quad\left|u_{\lambda}^{\prime}(\xi)\right| \leq K_{1} \widetilde{\varepsilon} \exp \left[-\vartheta\left(\xi-\xi_{0}\right)\right],
$$

for all $\xi \geq \xi_{0}$ and $\xi_{0}$ such that $\left|u_{\lambda}\left(\xi_{0}\right)-1\right| \leq \widetilde{\varepsilon}$. Moreover, the constants $K_{0}, K_{1}, \gamma>0$ and $\vartheta>0$ are independent of the solution $u_{\lambda}$.

PROOF. The system (3.1) can be written as a first order system. For $q \in[-Q, Q]$ all the eigenvalues of the linearized matrix for such a system at the points $\left(u, u^{\prime}\right)=(\mathbf{0}, \mathbf{0})$ and $(\mathbf{1}, \mathbf{0})$ have their real parts not equal to zero (see Theorem 3.3 in [5]). Now, the proof of Lemma 3 follows from the Hartman-Grobman theorem.

REMARK 3. Obviously the same estimates hold for the second derivatives of $u_{\lambda}^{\prime \prime}$, i.e. for some $K_{2}$ and all $\lambda \in[0,1]$,

$$
\left|u_{\lambda}^{\prime \prime}(\xi)\right| \leq K_{2} \widetilde{\varepsilon} \exp \left[\gamma\left(\xi-\xi_{0}\right)\right]
$$

for all $\xi \leq \xi_{0}$ and $\xi_{0}$ such that $\left|u_{\lambda}\left(\xi_{0}\right)\right| \leq \widetilde{\varepsilon}$, and

$$
\left|u_{\lambda}^{\prime \prime}(\xi)\right| \leq K_{2} \widetilde{\varepsilon} \exp \left[-\vartheta\left(\xi-\xi_{0}\right)\right]
$$

for all $\xi \geq \xi_{0}$ and $\xi_{0}$ such that $\left|u_{\lambda}\left(\xi_{0}\right)-1\right| \leq \widetilde{\varepsilon}$.

DEFINITION 2. Let $B_{2}$ denote the Banach space of functions $u: \mathbb{R}^{1} \rightarrow \mathbb{R}^{n}$ of $C^{2}\left(\mathbb{R}^{1}\right)$ class equipped with the norm

$$
\|u\|_{2}=\max _{i} \sup _{\xi}\left(\sum_{k=0}^{2}\left|u_{i}^{(k)}(\xi)\right|\right),
$$

with $u$ satisfying the following conditions:

$1^{o}$. the limits $\lim _{\xi \rightarrow \infty} u(\xi)$ and $\lim _{\xi \rightarrow-\infty} u(\xi)$ exist.

$2^{o} . u^{\prime}(\xi), u^{\prime \prime}(\xi) \rightarrow \mathbf{0}$ as $|\xi| \rightarrow \infty$.

Let $B_{20}$ denote the subspace of $B_{2}$ consisting of functions $u$ such that

$$
u_{1}(0)=\frac{1}{2} e_{1 *}\left(u_{1}(-\infty)+u_{1}(\infty)\right)
$$

where $e_{1 *}=\min _{J \in\{1, \ldots, K\}} e_{1}^{J}$.

Let $B_{0}$ denote the Banach space of functions $u: \mathbb{R}^{1} \rightarrow \mathbb{R}^{n}$ of $C^{0}\left(\mathbb{R}^{1}\right)$ class such that the limits $\lim _{\xi \rightarrow \infty} u(\xi)$ and $\lim _{\xi \rightarrow-\infty} u(\xi)$ exist, equipped with the norm

$$
\|u\|_{0}=\max _{i} \sup _{\xi}\left(\left|u_{i}(\xi)\right|\right) .
$$


Let

$$
\mathcal{M}(\lambda, q, u)=\left(\mathcal{M}_{1}, \ldots, \mathcal{M}_{n}\right),
$$

where $\mathcal{M}_{i}$ is defined in (3.2). The operator $\mathcal{M}$ acts from the space $\mathbb{R}^{1} \times \mathbb{R}^{1} \times B_{20}$ to the space $B_{0}$. It is Fréchet differentiable. (In particular its Fréchet derivative with respect to $(q, u)$ is continuous with respect to $(\lambda, q, u)$.) It is easy to check that the Fréchet derivative with respect to $(q, u)$ at the point $(\lambda, q, u)$ is the following operator

$$
D \mathcal{M}(\lambda, q, u)[\delta q, \delta u]=D_{u} \mathcal{M}(\lambda, q, u) \delta u+\mathcal{M}_{, q}(\lambda, q, u) \delta q,
$$

with

$$
D_{u} \mathcal{M}(\lambda, q, u) \delta u=\mathcal{A}\left(\lambda, u, u^{\prime}\right)(\xi)(\delta u)^{\prime \prime}+\mathcal{C}\left(\lambda, q, u, u^{\prime}, u^{\prime \prime}\right)(\xi)(\delta u)^{\prime}+\mathcal{B}\left(\lambda, q, u, u^{\prime}, u^{\prime \prime}\right)(\xi) \delta u,
$$

where

$$
\begin{gathered}
\mathcal{A}=\operatorname{diag}\left(a_{\lambda 1}\left(u_{1}, u_{1}^{\prime}\right), \ldots, a_{\lambda n}\left(u_{n}, u_{n}^{\prime}\right)\right)(\xi) \\
\mathcal{C}_{i j}=\left\{a_{\lambda i}\left(u_{i}, u_{i}^{\prime}\right) u_{i}^{\prime \prime}-(1-\lambda) q u_{i}^{\prime}+\lambda\left[-q c_{i}\left(u_{i}, u_{i}^{\prime}\right) u_{i}^{\prime}+M_{i}\left(u, u_{i}^{\prime}\right) u_{i}^{\prime}\right]\right\}_{, u_{j}^{\prime}}(\xi), \\
\mathcal{B}_{i j}=\left\{a_{\lambda i}\left(u_{i}, u_{i}^{\prime}\right) u_{i}^{\prime \prime}-\lambda q c_{i}\left(u_{i}, u_{i}^{\prime}\right) u_{i}^{\prime}+\lambda M_{i}\left(u, u_{i}^{\prime}\right) u_{i}^{\prime}+f_{i}(u)\right\}_{, u_{j}}(\xi)
\end{gathered}
$$

and

$$
\delta u=\left(\delta u_{1}, \ldots, \delta u_{n}\right)^{T} .
$$

Consider the linear operator:

$$
L u=\mathbf{A}(\xi) u^{\prime \prime}+\mathbf{C}(\xi) u^{\prime}+\mathbf{B}(\xi) u,
$$

where $\mathbf{A}(\xi), \mathbf{B}(\xi), \mathbf{C}(\xi)$ are matrices of $C^{1}$ class, $\mathbf{A}(\xi)$ and $\mathbf{C}(\xi)$ are diagonal matrices, $\mathbf{A}(\xi)$ has positive diagonal elements and $\mathbf{B}(\xi)$ has positive off-diagonal elements. Assume that the matrices $\mathbf{A}(\xi), \mathbf{B}(\xi), \mathbf{C}(\xi)$ have limits as $\xi \rightarrow \pm \infty$ and that the matrices $\mathbf{B}_{ \pm}=\lim _{\xi \rightarrow \pm \infty} \mathbf{B}(\xi)$ have negative principal eigenvalues.

The following result, which can be found in [16], will be of basic importance below.

LEMMA 4. (Proposition 1.3 p. 155 in [16]) Let us assume that a positive solution $w(\xi)$ exists for the equation

$$
L u=\mathbf{0},
$$

such that $\lim _{\xi \rightarrow \pm \infty} w(\xi)=0$. Then the following is true:

1) The equation 


$$
L u=\lambda u, \quad u( \pm \infty)=\mathbf{0}
$$

has no solutions different from $\mathbf{0}$ for $\operatorname{Re} \lambda \geq 0, \lambda \neq 0$.

2) Every solution of Eq. (3.7) has for $\lambda=0$ the form $u(\xi)=k w(\xi), k \in \mathbb{R}^{1}$.

3) The adjoint equation

$$
L^{*} v=\mathbf{0}, \quad v( \pm \infty)=\mathbf{0}
$$

has a positive solution. This solution is unique to within a constant factor.

Our starting point will be system (3.1) for $\lambda=0$. According to the results in [16], this system has a heteroclinic solution pair $\left(q_{0}, u_{0}(\xi)\right)$ with $u_{0} \in B_{20}$ and $u_{0}^{\prime}(\xi)>0$ for all $\xi \in \mathbb{R}^{1}$, joining the points $\mathbf{0}$ and 1. According to Lemma 4 there is a unique (up to a multiplicative constant) solution to the linearized system $D_{u} \mathcal{M}\left(0, q_{0}, u_{0}\right) \delta u=0$, namely $\delta u=u_{0}^{\prime}(\xi)$. In Lemma 5 we prove that the linearized operator $\operatorname{DM}\left(0, q_{0}, u_{0}\right)$ is boundedly invertible, i.e. the equation

$$
\operatorname{DM}\left(0, q_{0}, u_{0}\right)[\delta q, \delta u]=h,
$$

has a unique solution in the space $B_{20} \times \mathbb{R}^{1}$.

According to the implicit function theorem (see e.g. [3]) there exists $\lambda_{*}>0$ such that for all $\lambda \in\left[0, \lambda_{*}\right]$ there exists a heteroclinic pair for system (3.1). If $\lambda_{*}<1$ but $D \mathcal{M}\left(\lambda_{*}, u_{\lambda_{*}}, q_{\lambda_{*}}\right)[\delta q, \delta u]$ is boundedly invertible, then we can prolong the interval of existence of heteroclinics to $\left[0, \lambda_{* 1}\right]$, $\lambda_{* 1}>\lambda_{*}$. If this procedure can be repeated, then after a finite number of steps we are able to extend the existence interval to the whole of $[0,1]$.

LEMMA 5. Suppose that for $\lambda \in\left[0, \lambda_{b}\right], \lambda_{b} \in(0,1]$, there exists a heteroclinic pair $\left(q_{\lambda}, u_{\lambda}\right)$ satisfying the system (3.1), such that $u_{\lambda}$ is strictly monotonic in all of its components.

Then the linearized system

$$
\operatorname{DM}\left(\lambda, q_{\lambda}, u_{\lambda}\right)[\delta q, \delta u]=h,
$$

has for all $h \in B_{0}$ a unique (up to a multiplication constant) solution in the space $B_{20} \times \mathbb{R}^{1}$. The norm of $\left[\operatorname{DM}\left(\lambda, q, u_{\lambda}\right)\right]^{-1}$ is bounded uniformly by a constant independent of $\lambda \in\left[0, \lambda_{b}\right]$.

Before the proof of this lemma, let us note the following obvious fact.

LEMMA 6. Suppose that $\left(q_{\lambda}, u_{\lambda}(\xi)\right), \lambda \in\left[0, \lambda_{b}\right], \lambda_{b} \in[0,1]$, is a family of strictly monotone heteroclinic pairs (joining the points $\mathbf{0}$ and $\mathbf{1}$ ). Then:

1. For all natural $l \geq 1$ there exists $\varepsilon_{l}>0$, such that $u_{\lambda}^{\prime}(\xi)>\varepsilon_{l}$ for all $\xi \in[-l, l]$ and all $\lambda \in\left[0, \lambda_{b}\right]$.

2. For any $\varepsilon>0$ there exists $Z_{\varepsilon}>0$ such that $\left|u_{\lambda}(\xi)\right|<\varepsilon$ for all $\xi<-Z_{\varepsilon}$ and $\left|1-u_{\lambda}(\xi)\right|<\varepsilon$ for all $\xi>Z_{\varepsilon}$ for all $\lambda \in\left[0, \lambda_{b}\right]$. 
PROOF. Suppose that point 1. of the lemma is not true. Then there would exist $l \geq 1$, sequences $\left\{\lambda_{k}\right\}_{k=1}^{\infty},\left\{\varepsilon_{k}\right\}_{k=1}^{\infty}, \varepsilon_{k}>0, \lim _{k \rightarrow \infty} \varepsilon_{k}=0$, and an index $j \in\{1, \ldots, n\}$, such that $u_{\lambda_{k} j}^{\prime}\left(\xi_{k}\right) \leq \varepsilon_{k}$ for some $\xi_{k} \in[-l, l]$. Obviously, we may choose a convergent subsequence $\left\{\lambda_{m_{k}}\right\}_{k=1}^{\infty}$ of the sequence $\left\{\lambda_{k}\right\}_{k=1}^{\infty}$ such that $\lambda_{m_{k}} \rightarrow \lambda^{*}$ and $\xi_{m_{k}} \rightarrow \xi_{0} \in[-l, l]$. But this would mean that $\lim u_{\lambda^{*}}^{\prime}\left(\xi_{0}\right)=0$ contrary to the conditions of the lemma. Now, suppose that point 2 . of the lemma is not true for some $\varepsilon>0$ as $\xi \rightarrow \infty$ and there exists a sequence $\left\{\lambda_{k}\right\}_{k=1}^{\infty}$ and an index $j \in\{1, \ldots, n\}$ such that $u_{\lambda_{k} j}(\xi)<1-\varepsilon$ for all $\xi<k$. We may choose a convergent subsequence $\left\{\lambda_{m_{k}}\right\}_{k=1}^{\infty}$ of the sequence $\left\{\lambda_{k}\right\}_{k=1}^{\infty}$. If $\lambda_{m_{k}} \rightarrow \lambda^{*}$, then $u_{\lambda^{*} j}(\xi)<1-\varepsilon$ for all $\xi \in \mathbb{R}^{1}$. This is a contradiction with the fact that $\lim _{\xi \rightarrow \infty} u_{\lambda^{*} j}(\xi)=1$. Thus $u_{\lambda j}(\xi)>1-\varepsilon$ for all $\xi>Z_{\varepsilon+}$, where $Z_{\varepsilon+}>0$ is independent of $\lambda$. In the same way we may prove that there exists a number $Z_{\varepsilon-}>0$ such that $u_{\lambda j}(\xi)<\varepsilon$ for all $\xi<-Z_{\varepsilon-}$ and all $\lambda \in\left[0, \lambda_{b}\right]$. Taking $Z_{\varepsilon}=\max \left\{Z_{\varepsilon-}, Z_{\varepsilon+}\right\}$ proves the lemma.

The monotonicity property of the heteroclinic solutions to system (3.1) obtained by means of the implicit function theorem will be proved in Section 4.

PROOF OF LEMMA 5. First, we recall the basic facts from the theory of exponential dichotomy, which will be used below. We will use the results from [12] and [2].

The system

$$
x^{\prime}=S(\xi) x
$$

where $\xi \in \mathbb{R}^{1}, x \in C^{1}\left(\mathbb{R}^{1}, \mathbb{R}^{2 n}\right)$ and $S(\xi)$ is $2 n \times 2 n$ matrix, is said to have an exponential dichotomy on the half-line $\mathbb{R}_{+}^{1}$, if there exist a projection operator $\widetilde{P}: \mathbb{R}^{2 n} \rightarrow \mathbb{R}^{2 n}$, a multiplicative constant $\widetilde{L} \geq 1$ and an exponent constant $\beta>0$ such that for $s, t \in[0, \infty)$

$$
\left|X(t) \widetilde{P} X^{-1}(s)\right| \leq \widetilde{L} e^{-\beta(t-s)}
$$

for $s \leq t$, whereas

$$
\left|X(t)(I-\widetilde{P}) X^{-1}(s)\right| \leq \widetilde{L} e^{-\beta(s-t)}
$$

for $s \geq t$. Likewise, system (3.10) is said to have an exponential dichotomy on the half-line $\mathbb{R}_{-}^{1}$, if there exist a projection $\widetilde{Q}$, a multiplicative constant $\widetilde{L} \geq 1$ and an exponent constant $\beta>0$ such that for $s, t \in(-\infty, 0]$

$$
\left|X(t) \widetilde{Q} X^{-1}(s)\right| \leq \widetilde{L} e^{-\beta(t-s)}
$$

for $s \leq t$, whereas

$$
\left|X(t)(I-\widetilde{Q}) X^{-1}(s)\right| \leq \widetilde{L} e^{-\beta(s-t)}
$$

for $s \geq t$. Here || denotes a norm in the space of $2 n \times 2 n$ matrices, $X(\xi)$ is a fundamental matrix solution satisfying $X(0)=I$. For simplicity, we have taken the same constants for both of the half-lines.

System (3.9) is equivalent to the first order system

$$
x^{\prime}=A(\xi) x+\widetilde{h}(\xi),
$$


where $x=\left[\delta u_{1}, \ldots, \delta u_{n}, \delta u_{1}^{\prime}, \ldots, \delta u_{n}^{\prime}\right]^{T}$,

$$
A(\xi)=\left(\begin{array}{cc}
0 & I \\
\widetilde{\mathcal{B}}(\xi) & \widetilde{\mathcal{C}}(\xi)
\end{array}\right),
$$

with 0 and $I$ being the $n \times n$ zero and unit matrix respectively, $\widetilde{\mathcal{B}}(\xi)=\mathcal{A}^{-1}(\xi) \mathcal{B}(\xi), \widetilde{\mathcal{C}}(\xi)=$ $\mathcal{A}^{-1}(\xi) \mathcal{C}(\xi), \widetilde{h}_{k}(\xi) \equiv 0$ for $k=0, \ldots, n$, and

$$
\widetilde{h}_{k}(\xi)=h_{k-n}(\xi)+\left[(1-\lambda)+\lambda c_{k-n}\left(u_{\lambda(k-n)}(\xi), u_{\lambda(k-n)}^{\prime}(\xi)\right)\right] u_{\lambda(k-n)}^{\prime}(\xi) \delta q
$$

for $k=n+1, \ldots, 2 n$. If the matrix $A(\xi)$ was replaced by $A(\infty)$ or $A(-\infty)$ then the homogeneous system would have the property of exponential dichotomy (see [2] p. 10) on both of the sets $\mathbb{R}_{+}^{1}$ and $\mathbb{R}_{-}^{1}$ (as, due to Theorem 3.3 in [5], all the eigenvalues of both $A(-\infty)$ and $A(\infty)$ have nonzero real parts). It is clear that the constants $\widetilde{L}$ and $\beta$ may be chosen independent of $\lambda \in\left[0, \lambda_{b}\right]$. The projection operators may depend on $\lambda$, though, for simplicity, we will not denote it explicitly. Using the results of [2], one may prove that the property of exponential dichotomy takes place also for the full matrix $A(\xi)$ for both of the sets $\mathbb{R}_{+}^{1}$ and $\mathbb{R}_{-}^{1}$. Thus, let $\widetilde{\varepsilon}$ be the same as in Lemma 3 and Remark $3,0 \leq \varepsilon \leq \widetilde{\varepsilon}$, and $Z_{\varepsilon}$ such that $\left|u_{\lambda}(\xi)\right|<\varepsilon$ for $\xi<-Z_{\varepsilon},\left|1-u_{\lambda}(\xi)\right|<\varepsilon$ for $\xi>Z_{\varepsilon}$. All the possible values of heteroclinic parameters $q_{\lambda}$ are bounded in their absolute value by Lemma 3. If $\varepsilon \rightarrow 0$, then $\left|f_{i}\left(u_{\lambda}(\xi)\right)\right| \rightarrow 0, i \in\{1, \ldots, n\}$, for all $|\xi|>Z_{\varepsilon}$ hence using classical a priori estimates for second order elliptic equations on compact sets we conclude that also $\left|u_{\lambda}^{\prime}(\xi)\right| \rightarrow 0$ for all $|\xi|>Z_{\varepsilon}$. (On any interval $[k, k+1]$ with $k$ integer, we consider the Dirichlet problem with the right hand sides equal to $f_{i}\left(u_{\lambda}(\xi)\right)$.) Consequently, for $\varepsilon>0$ sufficiently small, $\max \left\{\sup _{\xi>Z_{\varepsilon}}|A(\xi)-A(\infty)|, \sup _{\xi<-Z_{\varepsilon}}|A(\xi)-A(-\infty)|\right\}<\beta\left(4 \widetilde{L}^{2}\right)^{-1}$. Let us fix $\varepsilon$ satisfying the above conditions and denote $Z_{\varepsilon}$ by $Z$. According to the results in [2] (see p. 13 and Proposition 1 in Chapter 6) system (3.11) satisfies the conditions of exponential dichotomy on both of the half-lines $\mathbb{R}_{-}^{1}$ and $\mathbb{R}_{+}^{1}$ with the exponent constant replaced by $\alpha=(\beta-2 K \delta)$ and the multiplicative constant replaced by $L=\frac{5}{2} \widetilde{L}^{2} \widetilde{N}^{2} k$, where $k=\exp (\alpha Z)$ and

$$
\widetilde{N}=\max \left\{\operatorname { e x p } \left(\int_{-Z}^{0}|A(s)| d s, \exp \left(\int_{0}^{Z}|A(s)| d s\right\} .\right.\right.
$$

Using Lemma 4.2 in [12] we conclude that the operator $x \rightarrow x^{\prime}-A(\xi) x$ is Fredholm as acting from the space $C^{1}\left(\mathbb{R}^{1}, \mathbb{R}^{2 n}\right)$ to the space $\left.C^{0}\left(\mathbb{R}^{1}, \mathbb{R}^{2 n}\right)\right)$. Note, that if $V$ is a bounded solution to the adjoint of the system $x^{\prime}=A(\xi) x$, i.e. the system $x^{\prime}=-A^{T}(\xi) x$, then $V(\xi)=[j(\xi), v(\xi)]$, where $v(\xi)$ is a solution to the equation $L^{*} v=0$. As $u_{\lambda}$ is strictly monotone, then from Lemma 4 we infer that $V$ is unique (up to a constant factor). According to Lemma 4.2 in [12] the necessary and sufficient condition for the existence of a solution to system (3.11) from the space $C^{1}\left(\mathbb{R}^{1}, \mathbb{R}^{2 n}\right)$ ) is the equality $\int_{-\infty}^{\infty} \sum_{i=1, \ldots 2 n} V_{i}(\xi) \widetilde{h}_{i}(\xi) d x=0$. Due to the form of $\widetilde{h}_{i}$ this condition is equivalent to the condition

$$
\int_{-\infty}^{\infty} \sum_{i=1, \ldots, n} v_{i}(\xi)\left[-\mathcal{M}_{i, q}\left(\lambda, q_{\lambda}, u_{\lambda}\right)(\xi) \delta q+h_{i}(\xi) d x\right]=0 .
$$

According to Lemma 4 the solution $v$ has all of its components positive. Moreover, all the components of $v$ must vanish exponentially, so, for a given $h$, both terms on the left hand side are finite. 
Consequently we have proved that $\delta q$ is uniquely determined by (3.13) and is a bounded function of $h$, i.e.

$$
|\delta q| \leq N_{q}|h|_{C^{0}\left(\mathbb{R}^{1}\right)}
$$

where $N_{q}$ is a constant independent of $\lambda \in\left[0, \lambda_{b}\right]$.

Given the value of $\delta q$, the general solution to system (3.9) has for $\xi \geq 0$ the form:

$$
\begin{gathered}
x(\xi)=c Y(\xi)+X(\xi) P X^{-1}(0) w+ \\
\int_{0}^{\xi} X(\xi) P X^{-1}(s) \widetilde{h}(s) d s-\int_{\xi}^{\infty} X(\xi)(I-P) X^{-1}(s) \widetilde{h}(s) d s
\end{gathered}
$$

and for $\xi \leq 0$ the form

$$
\begin{gathered}
x(\xi)=c Y(\xi)+X(\xi)(I-Q) X^{-1}(0) w+ \\
\int_{-\infty}^{\xi} X(\xi) Q X^{-1}(s) \widetilde{h}(s) d s-\int_{\xi}^{0} X(\xi)(I-Q) X^{-1}(s) \widetilde{h}(s) d s
\end{gathered}
$$

where $X(\xi)$ is a fundamental matrix solution, $w$ is a uniquely determined vector from $\mathbb{R}^{2 n}$ such that

$$
[P-(I-Q)] w=\int_{-\infty}^{0} Q X^{-1}(s) \widetilde{h}(s) d s+\int_{0}^{\infty}(I-P) X^{-1}(s) \widetilde{h}(s) d s
$$

which is orthogonal to all vectors $\eta$ satisfying the equation

$$
\eta^{*}[P-(I-Q)]=\mathbf{0} .
$$

As $u_{\lambda}^{\prime}(0)>\mathbf{0}$, the constant $c$ is uniquely determined by the vector $w$ and the condition $x_{1}(0)=$ $\frac{1}{2} e_{1 *}\left[x_{1}(-\infty)+x_{1}(\infty)\right]$. It easy to show that this solution tends to finite limits as $\xi \rightarrow \pm \infty$. Let us consider the case $\xi \rightarrow \infty$. Let $x_{+} \in \mathbb{R}^{2 n}$ be such that

$$
A(\infty) x_{+}+\widetilde{h}(\infty)=\mathbf{0}
$$

As $\widetilde{h}(\infty)=h(\infty)$ is well defined and $A(\infty)$ is nonsingular then $x_{+}$is well defined. The difference $\zeta(\xi)=x(\xi)-x_{+}$satisfies the vector equation

$$
\zeta^{\prime}-A(\xi) \zeta=(\widetilde{h}(\xi)-\widetilde{h}(\infty))+(A(\xi)-A(\infty)) x_{+} .
$$

The right hand side of this equation tends to $\mathbf{0}$ as $\xi$ tends to $\infty$. Thus applying (3.14) and using the property of exponential dichotomy we conclude that $\zeta(\xi) \rightarrow \mathbf{0}$ as $\xi \rightarrow \infty$. The same proof may be done for $\xi \rightarrow-\infty$. From the point of view of the second order system (3.9) it means that its solution (for the properly chosen $\delta q$ ) tends to finite limits and its first derivatives tend to $\mathbf{0}$ as $\xi \rightarrow \pm \infty$. Hence its second derivatives tend to $\mathbf{0}$. Hence any $C^{1}$ solution to (3.9) belongs to $B_{20}$. It is clear that $C^{2}\left(\mathbb{R}^{1}, \mathbb{R}^{n}\right)$ norm of the solution to system (3.9) is equivalent to the $C^{1}\left(\mathbb{R}^{1}, \mathbb{R}^{2 n}\right)$ norm of the corresponding solution to system (3.11) given by the above expressions. Given the value of $\delta q$, the last norm can be estimated by $C|\widetilde{h}|_{C^{0}\left(\mathbb{R}^{1}\right)}$, where $C$ is a constant. In fact, this constant depends on $u_{\lambda}$ only by the value of $Z$. According to the definition of $\widetilde{h}$, and using Lemmas 1 and 2 we conclude that 


$$
|\delta u|_{B_{20}} \leq C_{*}\left(1+N_{*} N_{q}\right)|h|_{B_{0}},
$$

where $C_{*}, N_{*}$ and $N_{q}$ are independent of $\lambda \in\left[0, \lambda_{b}\right]$. Now, we use Theorem 4.2-H p.180 in [14]. Namely, according to Lemmas $1,2,3,4$ and 6 , for each each $\lambda \in\left[0, \lambda_{b}\right]$ and for each $h \in B_{0}$ there exists a unique pair $[\delta q, \delta u] \in \mathbb{R}^{1} \times B_{20}$ satisfying system (3.9). Thus, using Theorem 4.2-H p.180 in [14], we infer that $D \mathcal{M}^{-1}$ is continuous hence it is bounded. Due to the compactness of the set $\left[0, \lambda_{b}\right]$ the operator $D \mathcal{M}^{-1}$ is uniformly bounded for all $\lambda \in\left[0, \lambda_{b}\right]$. The lemma is proved.

\section{Strict monotonicity of $u_{\lambda}$ and the existence proof}

In this section we demonstrate that the interval of $\lambda$ values, for which strictly monotone heteroclinic solutions exist can be extended to the whole of $[0,1]$. Roughly speaking the proof consists in showing that this interval is both relatively closed and open in $[0,1]$, so it must coincide with $[0,1]$.

In the previous section we showed that the operator $\mathcal{M}$ linearized around a heteroclinic pair $\left(q_{\lambda}, u_{\lambda}\right), \lambda \in[0,1]$ is boundedly invertible provided the function $u_{\lambda}$ is strictly monotone. For $\lambda=0$ system (3.1) takes the form:

$$
u_{i}^{\prime \prime}-q u_{i}^{\prime}+f_{i}(u)=0,
$$

$i=1, \ldots, n$. According to Theorem 1.1 p. 153 in [16] this system has a unique heteroclinic pair $\left(q_{0}, u_{0}\right)$ with $u_{0}$ is strictly monotone. The question arises, whether the solution may become non monotone for larger values of $\lambda$. First, we will show that if $u_{\lambda}$ is strictly monotone for $\lambda \in\left[0, \lambda_{0}\right)$ then it exists and is monotonic also for $\lambda=\lambda_{0}$.

LEMMA 7.Assume that $\left(q_{\lambda}, u_{\lambda}\right), \lambda \in\left[0, \lambda_{*}\right), \lambda_{*}>0$, is a continuous family of heteroclinic pairs (obtained by means of the implicit function theorem) and that $u_{\lambda}(\xi)$ is strictly monotonic for all $\lambda \in\left[0, \lambda_{0}\right), \lambda_{0} \in\left[0, \lambda_{*}\right]$. Then for $\lambda=\lambda_{0}$ the heteroclinic pair $\left(q_{\lambda_{0}}, u_{\lambda_{0}}(\xi)\right)$ also exists and $u_{\lambda_{0}}(\xi)$ is a strictly monotone function of $\xi$.

PROOF. Suppose contrary to the hypothesis of the lemma that there exists a sequence of $\left\{\lambda_{k}\right\}_{k=1}^{\infty}$ such that $\lambda_{k} \rightarrow \lambda_{0}$ and a sequence $\left\{\xi_{k}\right\}_{k=1}^{\infty}$ such that $\xi_{k} \rightarrow \xi_{0}$ as $k \rightarrow \infty$ such that $u_{\lambda_{k} j}^{\prime}\left(\xi_{k}\right) \rightarrow u_{\lambda_{0} j}^{\prime}\left(\xi_{0}\right)=0$ for some index $j$ and $u_{\lambda_{0} i}^{\prime}(\xi) \geq 0$ for all $\xi$ and all $i$. We will show that the sequence $\xi_{k}$ cannot be convergent to any finite $\xi_{0}$. Suppose to the contrary that $\left|\xi_{0}\right|<\infty$. First, let us assume that $\lambda_{0}<\lambda_{*}$. As $u_{\lambda_{0} j}^{\prime}$ attains a global minimum at $\xi_{0}$ then we have $u_{\lambda_{0} j}^{\prime \prime}\left(\xi_{0}\right)=$ $0, f_{j}\left(u_{\lambda_{0}}\left(\xi_{0}\right)\right)=0$ and $u_{\lambda_{0} j}^{\prime \prime \prime}\left(\xi_{0}\right) \geq 0$. Differentiating the $j$-th equation we obtain at the point $\xi_{0}$ :

$$
a_{\lambda_{0} j}\left(u_{\lambda_{0} j}, u_{\lambda_{0} j}^{\prime}\right) u_{\lambda_{0} j}^{\prime \prime \prime}+\sum_{i \neq j} f_{j, i} u_{\lambda_{0} i}^{\prime}=0
$$

There are two possibilities: $u_{\lambda_{0} i}^{\prime}\left(\xi_{0}\right)>0$ for some $i \neq j$ or $u_{\lambda_{0} i}^{\prime}\left(\xi_{0}\right)=0$ for al $i$. In the first case we arrive at contradiction due to the monotonicity conditions. In the second case we would have 
$u_{\lambda_{0} i}^{\prime \prime}\left(\xi_{0}\right)=0$ for all $i$ (by the same arguments as for the index $j$ ). In consequence $f\left(u\left(\xi_{0}\right)\right)=\mathbf{0}$ and the point $\left(u_{\lambda_{0}}\left(\xi_{0}\right), u_{\lambda_{0}}^{\prime}\left(\xi_{0}\right)\right)=\left(u_{\lambda_{0}}\left(\xi_{0}\right), \mathbf{0}\right)$ would be a singular point of the corresponding first order system. However, as the right hand sides of the considered system are of $C^{1}$ class, such a point could not be attained for finite $\xi_{0}$. So, let us assume that $\lambda_{0}=\lambda_{*}$. We will prove that for $\lambda=\lambda_{0}$ the heteroclinic pair to system (3.1) also exists. This can be done on the basis of Lemma 1 and Lemma 2. There exists a sequence $\left\{n_{k}\right\}_{k=1}^{\infty}$ such that $\left\{q_{\lambda_{k}}\right\}_{k=1}^{\infty}$ converges to some $q_{*}$. Out of this sequence we can in turn choose subsequences $\left\{n_{r k}\right\}_{k=1}^{\infty}$ having the property that the sequence $\left\{u_{\lambda_{n_{r k}}}\right\}$ converges to a function $u_{0 r}(\xi)$ uniformly in $\xi$ on the interval $[-r, r]$, where $r=1,2 \ldots$, and $\left\{n_{(r+1) k}\right\}_{k=1}^{\infty}$ is a subsequence of $\left\{n_{r k}\right\}_{k=1}^{\infty}$. (The convergence is guaranteed by Arzeli lemma and bootstrap argument.) Hence by choosing a diagonal subsequence we obtain a sequence $\left\{m_{k}\right\}_{k=1}^{\infty}$ such that $\left\{u_{\lambda_{m_{k}}}\right\}_{k=1}^{\infty}$ converges to a function $u_{*}(\xi)$ uniformly in $\xi$ on every interval $[-r, r]$. Now, due to the strict monotonicity of $u_{\lambda_{k}}(\xi)$ the function $u_{*}(\xi)$ is monotone. Hence it must have finite limits and $u_{0}^{\prime}(\xi) \rightarrow \mathbf{0}$ as $\xi \pm \infty$. So, for all $i$, we have $u_{* i}^{\prime \prime}(\xi) \rightarrow f_{i}\left(u_{*}( \pm \infty)\right)=$ const as $\xi \rightarrow \pm \infty$. It follows that $u_{* i}^{\prime \prime}(\xi) \rightarrow 0$ as $\xi \pm \infty$. So both $u_{*}(-\infty)$ and $u_{*}(\infty)$ must belong to the set $\left\{\mathbf{0}, E_{\lambda_{0}}^{1}, \ldots, E_{\lambda_{0}}^{K}, \mathbf{1}\right\}$. Due to the condition satisfied by $u_{\lambda_{k} 1}(0)$ we conclude that $u_{*}(\xi) \rightarrow \mathbf{0}$ as $\xi \rightarrow-\infty$. Due to the monotonicity of $u_{*}$ we conclude that $u_{*}(\infty)=E^{J}$, where $J \in\{1, \ldots, n\}$ or $u_{*}(\infty)=1$. The first possibility cannot take place, because this would mean that the heteroclinic solution splits into two heteroclinics as $\lambda_{m_{k}} \rightarrow \lambda_{0}$ : one joining the points $\mathbf{0}$ and $E^{J}$ and the other joining the points $E^{J}$ and 1. (Note that, according to Theorem 2.5 in [5] a monotone heteroclinic solution cannot join the singular points $\left(E_{\lambda_{0}}^{J_{1}}, \mathbf{0}\right)$ and $\left(E_{\lambda_{0}}^{J_{2}}, \mathbf{0}\right)$ with $J_{1} \neq J_{2}$.) Let us sketch the proof of this fact. Let $s_{k}>0$ be such that $u_{\lambda_{k} 1}\left(s_{k}\right)=\frac{1}{2}\left(1+e_{1}^{J}\right)$. Then out of the sequence $\left\{u_{\lambda_{m_{k}}}\right\}_{k=1}^{\infty}$ we may create a sequence of functions $U_{\lambda_{m_{k}}}(\xi)=u_{\lambda_{m_{k}}}\left(\xi+s_{m_{k}}\right)$. As before, due to Lemmas 1 and 2 we may find a subsequence of $\left\{m_{k}\right\}_{k=1}^{\infty}$ (denoted for simplicity in the same way) such that $q_{\lambda_{m_{k}}} \rightarrow q_{*}$ and $U_{\lambda_{m_{k}}}(\xi) \rightarrow U_{*}(\xi)$ on every bounded interval of the form [-r, $\left.r\right]$. Obviously, $s_{m_{k}} \rightarrow \infty$. Moreover, if $\left\{\xi_{m_{k}}\right\}_{k=1}^{\infty}$ is the sequence of points such that $u_{\lambda_{m_{k}}}\left(\xi_{m_{k}}\right) \rightarrow E^{J}$ as $k \rightarrow \infty$, then we have $s_{k}-\xi_{k} \rightarrow \infty$. Suppose that it is not true. Then (by taking a subsequence if necessary) $\xi_{m_{k}}-s_{m_{k}}$ would converge as $k \rightarrow \infty$ to some finite number $S<0$. It would mean however that $\lim _{k \rightarrow \infty} U_{\lambda_{m_{k}}}\left(\xi_{m_{k}}-s_{m_{k}}\right)=U_{*}(S)=\lim _{k \rightarrow \infty} u_{\lambda_{m_{k}}}\left(\xi_{m_{k}}\right)=u_{\lambda_{0}}(\infty)=E^{J}$. Thus $\left(U_{\lambda_{0}}(\xi), U_{\lambda_{0}}^{\prime}(\xi)\right)$ would attain the singular point $\left(E^{J}, \mathbf{0}\right)$ in a finite time. This is impossible. Hence $U_{*}(-\infty)=\lim _{k \rightarrow \infty} U_{\lambda_{m_{k}}}\left(\xi_{m_{k}}-s_{m_{k}}\right)=\lim _{k \rightarrow \infty} u_{\lambda_{m_{k}}}\left(\xi_{m_{k}}\right)=E^{J}$. In the same way $U_{0}^{\prime}(-\infty)=\lim _{k \rightarrow \infty} U_{\lambda_{m_{k}}}^{\prime}\left(\xi_{m_{k}}-s_{m_{k}}\right)=\lim _{k \rightarrow \infty} u_{\lambda_{m_{k}}}^{\prime}\left(\xi_{m_{k}}\right)=\mathbf{0}$. Due to the condition imposed on $s_{m_{k}}$ we also have $U_{\lambda_{m_{k}}}(\xi) \rightarrow \mathbf{1}$ as $\xi \rightarrow \infty$. In consequence for the same value of $q=q_{*}$ we would thus have two solutions $u_{*}$ and $U_{*}$ with positive first derivative attaining the point $\left(E^{J}, \mathbf{0}\right)$ as $\xi$ tends to $\infty$ and $-\infty$ respectively. This is however impossible due to a modification of the proof Lemma 2.4 p.161 in [16] (see also Lemma 3.8 in [4]). Thus the second possibility takes place and for $\lambda=\lambda_{0}$ the heteroclinic pair $\left(q_{*}, u_{*}\right)(\xi)$ exists. Hence repeating the arguments from the first part we conclude that $u_{0}(\xi)$ is strictly monotone.

LEMMA 8. Assume that $\left(q_{\lambda}, u_{\lambda}\right), \lambda \in\left[0, \lambda_{*}\right), \lambda_{*}>0$, be a continuous family of heteroclinic pairs (obtained by means of the implicit function theorem) and that $u_{\lambda} \in B_{20}$ is strictly monotonic for all $\lambda \in\left[0, \lambda_{0}\right], \lambda_{0} \in\left[0, \lambda_{*}\right)$. Then $u_{\lambda}$ is also a strictly monotonic for all $\lambda \geq \lambda_{0}$ sufficiently close to it.

PROOF. Suppose to the contrary that there exists a sequence $\left\{\lambda_{k}\right\}_{k=1}^{\infty}$ such that $\lambda_{k}>\lambda_{0}$ for 
all $k, \lim _{k \rightarrow \infty} \lambda_{k}=\lambda_{0},\left(q_{\lambda_{k}}, u_{\lambda_{k}}\right)$ is a heteroclinic pair satisfying the system (3.1) for $\lambda=\lambda_{k}$ and $u_{\lambda_{k}}$ are not strictly monotonic functions tending to $u_{\lambda_{0}}$, i.e.

$$
\left\|u_{\lambda_{k}}-u_{\lambda_{0}}\right\|_{C^{2}\left(\mathbb{R}^{1}\right)} \rightarrow 0, \quad\left|q_{\lambda_{k}}-q_{\lambda_{0}}\right| \rightarrow 0
$$

as $k \rightarrow \infty$. As $u_{\lambda_{k}}$ are not strictly monotonic, then there exist sequences $\left\{\xi_{k}\right\}_{k=1}^{\infty}$ and $j(k)$ such that $u_{\lambda_{k} j}^{\prime}\left(\xi_{k}\right) \leq 0$. Due to (4.2) there must exist a subsequence of $\left\{\xi_{k}\right\}_{k=1}^{\infty}$, which we will denote also by $\left\{\xi_{k}\right\}_{k=1}^{\infty}$, converging to some finite $\xi_{0}$, such that $u_{\lambda_{0} j}^{\prime}\left(\xi_{0}\right)=0$. If $\left|\xi_{0}\right|$ is finite, then we would arrive at contradiction as we would have $u_{\lambda_{0}}^{\prime}\left(\xi_{0}\right) \leq \mathbf{0}$ and $u_{\lambda_{0}}^{\prime}\left(\xi_{0}\right)$ is positive according to the proof Lemma 7. Now, suppose that the subsequence of $\left\{\xi_{k}\right\}_{k=1}^{\infty}$ converges to $\xi_{0}=-\infty$ or $\xi_{0}=\infty$. We will consider only the case $\xi_{0}=\infty$. Obviously, according to the definition of the space $B_{20}$, $u_{\lambda_{k}}\left(\xi_{k}\right) \rightarrow \mathbf{1}, u_{\lambda_{k}}^{\prime}\left(\xi_{k}\right) \rightarrow \mathbf{0}$ and $u_{\lambda_{k}}^{\prime \prime}\left(\xi_{k}\right) \rightarrow \mathbf{0}$ as $k \rightarrow \infty$. Let $l$ and $\xi^{*}$ be such that for all $k \geq l$ we have $\xi_{k}>\xi^{*}, u_{\lambda_{k}}^{\prime}\left(\xi^{*}\right)>0$ and the matrices

$$
\begin{gathered}
\mathcal{B}_{i j}\left(\lambda_{k}, \xi\right)=\left\{a_{\lambda i, u_{j}}\left(u_{\lambda_{k} i}(\xi), u_{\lambda_{k} i}^{\prime}(\xi)\right) u_{\lambda_{k} i}^{\prime \prime}(\xi)-\lambda_{k} q c_{i, u_{j}}\left(u_{i}, u_{i}^{\prime}\right) u_{i}^{\prime}\right. \\
\left.+\lambda_{k} M_{i, u_{j}}\left(u_{\lambda_{k}}(\xi), u_{\lambda_{k} i}^{\prime}(\xi)\right) u_{\lambda_{k} i}^{\prime}(\xi)\right\}+f_{i, u_{j}}\left(u_{\lambda_{k}}(\xi)\right) .
\end{gathered}
$$

satisfy for all $\xi \geq \xi^{*}$ the following conditions:

1. $D \mathcal{B}\left(\lambda_{k}, \xi\right)$ has all of its off-diagonal terms positive,

2. $D \mathcal{B}\left(\lambda_{k}, \xi\right) P<\mathbf{0}$ where $P$ is a positive eigenvector corresponding to the principal eigenvalue of the matrix $D f(\mathbf{1})$.

Let us fix $k>l$. The vector function $v(\xi)=u_{\lambda_{k}}^{\prime}(\xi)$ satisfies the system:

$$
\begin{gathered}
a_{\lambda i}\left(u_{\lambda_{k} i}(\xi), u_{\lambda_{k} i}^{\prime}(\xi)\right) v_{i}^{\prime \prime}(\xi)+T_{i}\left(\lambda_{k}, q, \xi\right) v_{i}^{\prime}(\xi)+\sum_{j} \mathcal{B}_{i j}\left(\lambda_{k}, \xi\right) v_{j}(\xi)=0, \\
v\left(\xi^{*}\right)=u_{\lambda_{k}}^{\prime}\left(\xi^{*}\right), \quad v(\infty)=0,
\end{gathered}
$$

$i \in\{1, \ldots, n\}$, obtained by differentiation of system (3.1). Now, one may prove that the supposition $u_{\lambda_{k} j(k)}^{\prime}\left(\xi_{k}\right) \leq 0$ leads to a contradiction. The proof is carried out as the proof of Proposition 1.2 p.154 in [16] and we will not repeat it here.

LEMMA 9. The family $\left(q_{\lambda}, u_{\lambda}\right)$ of strictly monotone heteroclinic pairs can be continued at least till $\lambda=1$.

PROOF. Suppose that there exists $\lambda_{c r} \leq 1$ such that we cannot prolong the family of solutions beyond $\lambda_{c r}$ or just up to it. First, as in the proof of Lemma 7 we can prove that the heteroclinic pair $\left(q_{\lambda_{c r}}, u_{\lambda_{c r}}\right)$ exists and it is strictly monotonic. Using Lemma 6 and Lemma 5 we conclude that the linearized operator $\operatorname{DM}\left(\lambda_{c r}, q_{\lambda_{c r}}, u_{\lambda_{c r}}\right)$ has a bounded inverse. Thus, the family of solutions pairs $\left(q_{\lambda}, u_{\lambda}\right) \in \mathbb{R}^{1} \times B_{20}$ to system (3.1) could be prolonged beyond the value $\lambda_{c r}$, say to an interval $\left[0, \lambda_{c r}+\delta\right]$ with $\delta>0$ sufficiently small. Due to the implicit function theorem

$$
\left\|u_{\lambda_{1}}-u_{\lambda_{2}}\right\|_{B_{20}} \rightarrow 0 \quad \text { as } \quad \lambda_{2} \rightarrow \lambda_{1}
$$

for all $\lambda_{1}, \lambda_{2} \in\left[\lambda_{c r}, \lambda_{c r}+\delta\right]$. Now, let us note, that the singular points $\mathbf{0}$ and $\mathbf{1}$ are isolated independently of $q$ and $\lambda$, so the limits at $\pm \infty$ of $u_{\lambda_{1}}$ and $u_{\lambda_{2}}$ must be the same, if only $\left|\lambda_{2}-\lambda_{1}\right|$ is taken sufficiently small. Starting from $\lambda=\lambda_{c r}$ we conclude that, for all $\lambda \in\left[\lambda_{c r}, \lambda_{c r}+\delta\right],\left(q_{\lambda}, u_{\lambda}(\xi)\right)$ are 
heteroclinic pairs in the sense of Definition 1 . Using Lemma 8 we infer that $u_{\lambda}$ is strictly monotone for all $\lambda>\lambda_{c r}$ sufficiently close to it. But this is a contradiction to our supposition.

We are thus in a position to formulate the main theorem of our paper.

THEOREM 1. Let assumptions of Section 2 be satisfied. Then there exists a family of heteroclinic pairs $\left(q_{\lambda}, u_{\lambda}\right) \in \mathbb{R}^{1} \times C^{2}\left(\mathbb{R}^{1}\right), \lambda \in[0,1]$, such that each $\left(q_{\lambda}, u_{\lambda}\right)$ is a unique (up to translation in $\xi$ ) solution to system (3.1), $u_{\lambda}(-\infty)=\mathbf{0}, u_{\lambda}(\infty)=\mathbf{1}$ and $u_{\lambda}^{\prime}(\xi)>\mathbf{0}$ for $x \in \mathbb{R}^{1}$. This family is continuous, i.e. for all $\lambda_{1}, \lambda_{2} \in[0,1]$ :

$$
\left|q_{\lambda_{1}}-q_{\lambda_{2}}\right|+\left\|u_{\lambda_{1}}-u_{\lambda_{2}}\right\|_{B_{20}} \rightarrow 0 \quad \text { as } \quad \lambda_{2} \rightarrow \lambda_{1} \text {. }
$$

In particular $(q, u)=\left(q_{1}, u_{1}\right)$ is a heteroclinic pair for system (1.3) joining the points $\mathbf{0}$ and $\mathbf{1}$.

PROOF. Existence of $\left(q_{\lambda}, u_{\lambda}\right)$ follows from Lemma 9. Let us prove the uniqueness of the pair. First, the pair $\left(q_{0}, u_{0}\right)$ is unique. Suppose to the contrary that for some $\eta \in[0,1]$ we have at least two heteroclinic pairs $\left(q_{\eta i}, u_{\eta i}\right), i=1,2$. These solutions can be continued back to the value $\lambda=0$, so there must exist $\eta_{0}$ such that for $\lambda=\eta_{0}$ these two solutions merge for the first time, i.e. $\left(q_{\lambda 1}, u_{\lambda 1}\right) \neq\left(q_{\lambda 2}, u_{\lambda 2}\right)$ for all $\lambda \in\left(\eta_{0}, \eta\right]$. But, then due to the implicit function theorem we would have also $\left(q_{\lambda 1}, u_{\lambda 1}\right)=\left(q_{\lambda 2}, u_{\lambda 2}\right)$ for all $\lambda$ in some vicinity of $\eta_{0}$. This is a contradiction, from which the uniqueness follows.

\section{Generalization}

Existence of waves can be proved for more general classes of systems. Instead of the condition (2.2) we introduce the following assumption.

ASSUMPTION 1. For each $i \in\{1, \ldots, n\}$ one of the following conditions holds:

1. For all $u_{i} \in[0,1]$ and all $z_{i} \in \mathbb{R}_{+}^{1}$ either $a_{i, u_{i}}\left(u_{i}, z_{i}\right) \leq 0$ or $a_{i, u_{i}}\left(u_{i}, z_{i}\right) \geq 0$. The function $M_{i}\left(u, z_{i}\right)$ satisfies the estimation:

$$
\left|M_{i}\left(u, z_{i}\right)\right| \leq k(|u|)\left(1+\beta_{i}\left(\left|z_{i}\right|\right)\right),
$$

with $k: \mathbb{R}_{+}^{1} \rightarrow \mathbb{R}_{+}^{1}$ continuous, and $\beta_{i}: \mathbb{R}_{+}^{1} \rightarrow \mathbb{R}_{+}^{1}$ continuous and such that $\beta_{i}(y) y\left(\chi_{i}(y)\right)^{-1} \rightarrow 0$ as $y \rightarrow \infty$.

2. $c_{i} \equiv 1$ and for all $p, r \in \mathbb{R}_{+}^{1}, p \leq r$,

$$
M_{i}(u, p) \geq M_{i}(v, r)-\hat{M}_{i}(u, p, v, r)
$$

for all $\mathbf{0} \leq u \leq v \leq \mathbf{1}, \hat{M}_{i}(u, p, v, r) \leq k(u, v)\left(1+\beta_{i}(|r|)\right)$, with $k: \mathbb{R}_{+}^{2 n} \rightarrow \mathbb{R}_{+}^{1}$ continuous, $\beta_{i}: \mathbb{R}_{+}^{1} \rightarrow \mathbb{R}_{+}^{1}$ continuous and such that $\beta_{i}(y) y\left(\chi_{i}(y)\right)^{-1} \rightarrow 0$ as $y \rightarrow \infty$. 
3. $c_{i} \equiv 1$ and for all $p, r \in \mathbb{R}_{+}^{n}, p \leq r$,

$$
M_{i}(u, p) \leq M_{i}(v, r)+\hat{M}_{i}(u, p, v, r)
$$

for all $\mathbf{0} \leq v \leq u \leq \mathbf{1}, \hat{M}_{i}(u, p, v, r) \leq k(u, v)\left(1+\beta_{i}(|r|)\right)$, with $k: \mathbb{R}_{+}^{2 n} \rightarrow \mathbb{R}_{+}^{1}$ continuous, $\beta_{i}: \mathbb{R}_{+}^{1} \rightarrow \mathbb{R}_{+}^{1}$ continuous and such that $\beta_{i}(y) y\left(\chi_{i}(y)\right)^{-1} \rightarrow 0$ as $y \rightarrow \infty$.

4. $a_{i}\left(u_{i}, z_{i}\right)=a_{i}\left(u_{i}\right)$ and $M_{i}\left(u, z_{i}\right)$ satisfies condition (5.1) with $\beta_{i}(y) y^{-1} \rightarrow 0$ as $y \rightarrow \infty$, or the sum $a_{i}\left(u_{i}\right) u_{i}^{\prime \prime}+M_{i}\left(u, u_{i}^{\prime}\right) u_{i}^{\prime}$ can be written in the form $\left(a_{i}\left(u_{i}\right) u_{i}^{\prime}\right)^{\prime}+\mu_{i}\left(u, u_{i}^{\prime}\right) u_{i}^{\prime}$, where $\mu_{i, u_{j}}\left(u, z_{i}\right)>0$ for all $j \neq i$ and $u$ from some open neighbourhood of the set $[0,1]^{n}$, and $\mu_{i}\left(u, z_{i}\right)$ satisfies (5.1) with $\beta_{i}(y) y^{-1} \rightarrow 0$ as $y \rightarrow \infty$.

Points 2. and 3. of Assumption 1 are taken from paper [4]. Let us note that in this case we do not assume any growth condition on the term $M_{i}$. Let us emphasize that for different $i \in\{1, \ldots, n\}$ different conditions out of 1.,2.,3.,4. may be satisfied.

Lemma 1 and Lemma 2 may be proved also, when Assumption 1 is satisfied instead (2.2). The proofs are given in [8]. Thus the following theorem holds.

THEOREM 2. Let Assumption 1 be satisfied. Then there exists a family of heteroclinic pairs $\left(q_{\lambda}, u_{\lambda}\right) \in \mathbb{R}^{1} \times C^{2}\left(\mathbb{R}^{1}\right), \lambda \in[0,1]$, such that each $\left(q_{\lambda}, u_{\lambda}\right)$ is a unique (up to translation in $\xi$ ) solution to system (3.1), $u_{\lambda}(-\infty)=\mathbf{0}, u_{\lambda}(\infty)=\mathbf{1}$ and $u_{\lambda}^{\prime}(\xi)>\mathbf{0}$ for $x \in \mathbb{R}^{1}$. This family is continuous, i.e. for all $\lambda_{1}, \lambda_{2} \in[0,1]$ :

$$
\left|q_{\lambda_{1}}-q_{\lambda_{2}}\right|+\left\|u_{\lambda_{1}}-u_{\lambda_{2}}\right\|_{B_{20}} \rightarrow 0 \quad \text { as } \quad \lambda_{2} \rightarrow \lambda_{1} .
$$

In particular $(q, u)=\left(q_{1}, u_{1}\right)$ is a heteroclinic pair for system (1.3) joining the points $\mathbf{0}$ and $\mathbf{1}$.

Point 1. of Assumption 1 may be still generalized to points (a) and (b) of Assumption 4 in [8], but for the sake of simplicity we will not consider it here.

\section{Self contained proof of existence}

In this section we will show that if there exists an appropriate homotopy between $f$ and a bistable symmetric vector function $g$, then we can prove the existence of monotone heteroclinic solutions without referring to the results concerning the existence for the system with constant coefficients.

DEFINITION 3. Let $g(u)=\left(g_{1}(u), \ldots, g_{n}(u)\right)$ denote a $C^{1}\left(\mathbb{R}^{n}\right)$ function satisfying for all $i \in\{2, \ldots, n\}$ the following conditions:

1. $g_{i}\left(u_{1}, u_{2}, \ldots, u_{i-1}, u_{i}, u_{i+1}, \ldots, u_{n}\right)=g_{1}\left(u_{i}, u_{2}, \ldots, u_{i-1}, u_{1}, u_{i+1}, \ldots, u_{n}\right)$,

2. $g_{1,1}(u) \leq-k, g_{1, i}(u) \geq k$ for all $u \in \mathbb{R}^{n}, i \in\{2, \ldots, n\}, k>0$.

3. $\sum_{i=1}^{n} g_{1, i}(\mathbf{0})<0, \sum_{i=1}^{n} g_{1, i}(\mathbf{1})<0$. 
4. the only solutions to the equation $g\left(u_{1}, u_{1}, \ldots, u_{1}\right)=\mathbf{0}$ are $\mathbf{0}, \mathbf{1}$ and $E_{0}=\left(e_{01}, \ldots, e_{0 n}\right)$.

The following lemma holds.

LEMMA 10. The solutions to the equation $g(u)=\mathbf{0}$ must lie on the diagonal of $\mathbb{R}^{n}$.

ASSUMPTION 2. There exists a function

$$
G_{\lambda}(u):[0,1] \times \mathbb{R}^{n}
$$

such that

$$
G_{1}(u) \equiv f(u), \quad G_{0}(u) \equiv g(u) .
$$

Moreover for all $\lambda \in[0,1], G_{\lambda}(\mathbf{0})=G_{\lambda}(\mathbf{1})=0$. There is exactly one solution $E_{\lambda}=\left(e_{\lambda 1}, \ldots, e_{\lambda n}\right) \in$ $(0,1)^{n}$ to the system $G_{\lambda}(u)=\mathbf{0}$ different from $\mathbf{0}$ and $\mathbf{1}$. The matrices $D G_{\lambda}(\mathbf{0})$ and $D G_{\lambda}(\mathbf{1})$ have all eigenvalues in the left-half plane, and the principal eigenvalue of the matrix $D G_{\lambda}\left(E_{\lambda}\right)$ is positive.

An example of such a transformation will be given in Section 7 .

We should take into account that the nonlinear source function also changes with $\lambda$. Thus instead of (3.1) we consider the system of the same form but with different $\mathcal{M}_{i}$. Namely

$$
\mathcal{M}_{i}(\lambda, q, u)=a_{\lambda i}\left(u_{i}, u_{i}^{\prime}\right) u_{i}^{\prime \prime}-(1-\lambda) q u_{i}^{\prime}+\lambda\left[-q c_{i}\left(u_{i}, u_{i}^{\prime}\right) u_{i}^{\prime}+M_{i}\left(u, u_{i}^{\prime}\right) u_{i}^{\prime}\right]+G_{\lambda i}(u) .
$$

For $\lambda=0$ system (3.1) takes the form

$$
u_{i}^{\prime \prime}-q u_{i}^{\prime}+g_{i}(u)=0,
$$

$i=1, \ldots, n$. According to (Lemma 3.2 p.173 in [16]) to each bounded $C^{2}$ solution of the equation

$$
v^{\prime \prime}-q v^{\prime}+g(v, \ldots, v)=0,
$$

such that $v(-\infty)=0$ and $v(\infty)=1$ there corresponds a solution

$$
u(\xi)=(v(\xi), \ldots, v(\xi))
$$

to problem (6.4) and each solution to this problem has the form (6.4), where $v(\xi)$ satisfies (6.5). Thus there exists a unique strictly monotone heteroclinic pair $\left(q_{0}, u_{0}\right)$ satisfying system (6.4).

The whole proof of existence may be repeated almost verbatim, though now we must write everywhere $G_{\lambda i}$ instead of $f_{i}$. Also the definition of the space $B_{20}$ must be adjusted. Namely we take

$$
e_{1 *}=\min _{\lambda \in[0,1]} e_{\lambda 1} .
$$

The details are left to the reader. We have thus proved 
THEOREM 3. Let Assumptions 1 and 2 be satisfied. Then there exists a family of heteroclinic pairs $\left(q_{\lambda}, u_{\lambda}\right) \in \mathbb{R}^{1} \times C^{2}\left(\mathbb{R}^{1}\right), \lambda \in[0,1]$, such that each $\left(q_{\lambda}, u_{\lambda}\right)$ is a unique (up to translation in $\xi$ ) solution to system (3.1) (with $\mathcal{M}_{i}$ given by (6.3)), $u_{\lambda}(-\infty)=\mathbf{0}, u_{\lambda}(\infty)=\mathbf{1}$ and $u_{\lambda}^{\prime}(\xi)>\mathbf{0}$ for $x \in \mathbb{R}^{1}$. This family is continuous, i.e. for all $\lambda_{1}, \lambda_{2} \in[0,1]$ :

$$
\left|q_{\lambda_{1}}-q_{\lambda_{2}}\right|+\left\|u_{\lambda_{1}}-u_{\lambda_{2}}\right\|_{B_{20}} \rightarrow 0 \quad \text { as } \quad \lambda_{2} \rightarrow \lambda_{1} .
$$

In particular $(q, u)=\left(q_{1}, u_{1}\right)$ is a heteroclinic pair for system (1.3) joining the points $\mathbf{0}$ and $\mathbf{1}$.

It is also obvious that the same continuity result holds, when we can make a homotopy between $f$ and a function $g$ satisfying Assumption 2, in such a way that the number and the properties of its unstable zeros do not change.

\section{Application to multicomponent plasma}

Let us consider a system of equations describing multicomponent plasma sustained by a laser beam of a given intensity $I$. By this we mean plasma created in gas consisting of $(n-1) \geq 1$ different components. Under a constant pressure $p$ the temperatures $T_{1}$ of the light (electron) component and the temperatures of $T_{i}, i \in\{2, \ldots, n\}$ of heavy particles (atoms and ions) of $i$-th kind are described by the following equations (see [6],[7],[13],[9],[10]):

$$
\begin{gathered}
\left(\frac{\partial}{\partial t}+\vec{v}_{1} \cdot \nabla\right)\left\{\frac{3}{2} k_{B} N_{1} T_{1}+\widetilde{E}\left(T_{1}\right)\right\}=\nabla\left(k_{1} \nabla T_{1}\right)+f_{1}(T) \\
\left(\frac{\partial}{\partial t}+\vec{v}_{i} \cdot \nabla\right)\left\{\frac{3}{2} k_{B} N_{i} T_{i}\right\}=\nabla\left(k_{i} \nabla T_{i}\right)+f_{i}(T),
\end{gathered}
$$

where $i \in\{2, \ldots, n\}, T=\left(T_{1}, \ldots, T_{n}\right), k_{j}=k_{j}\left(T_{j}\right), j \in\{1, \ldots, n\}$, is the heat conductivity coefficient, $N_{1}\left(T_{1}\right)$ is the number density of electrons, $N_{i}\left(T_{i}\right), i \in\{2, \ldots, n\}$ is the number density of the heavy component of $i$-th kind and $\vec{v}_{j}(T), j \in\{1, \ldots, n\}$, denotes the convectional velocity of the $j$-th component, $k_{B}$ is the Boltzmann constant, $\widetilde{E}\left(T_{1}\right)$ is the average ionization energy for the given temperature $T_{1}$. (The energy necessary to the first ionization of an atom depends on the kind of the atom. If we have to deal with a one-component plasma, then $\widetilde{E}$ would be equal simply to $N_{1}\left(T_{1}\right) E$, where $E$ is the first ionization energy for the given kind of atoms.) The functions $f_{i}$ have the following form:

$$
\begin{array}{r}
f_{1}=F_{1}\left(T_{1}\right)+\sum_{j \in\{2, \ldots, n\}} c_{1 j}(T)\left(T_{j}-T_{1}\right) \\
f_{i}=\sum_{j \in\{1, \ldots, n\}, j \neq i} c_{i j}(T)\left(T_{j}-T_{i}\right)+K_{i}(T),
\end{array}
$$

for $i=2, \ldots, n$. The term $F_{1}=\kappa\left(T_{1}\right) I-\mathcal{E}_{\text {rad }}\left(T_{1}\right)$ is responsible for the absorption of energy from the laser beam $(\kappa I)$ and its losses by radiation $\left(\mathcal{E}_{\text {rad }}\right)$. The terms $K_{i}(T)$ describe the losses of energy in the process of heat conduction and convection. The terms $c_{i j}(T)\left(T_{j}-T_{i}\right)$ describe the transfer of energy from the $i$-th to the $j$-th component of the plasma.

Let us look for solutions in the form of travelling waves:

$$
T_{i}(x, t)=u_{i}(x \cdot \vec{n}+q t), \quad i=1, \ldots, n,
$$


where $\vec{n} \in \mathbb{R}^{3}$ is a chosen unit vector (a direction of propagation) and $\chi \in \mathbb{R}^{1}$ is the speed of the wave. If we denote $\xi:=x \cdot \vec{n}+\chi t$, then we arrive at a system of ordinary differential equations:

$$
\left(k_{i} u_{i}^{\prime}\right)^{\prime}-q C_{i}\left(u_{i}\right) u_{i}^{\prime}-\vec{v}_{i} \cdot \vec{n} C_{i}\left(u_{i}\right) u_{i}^{\prime}+f_{i}(u)=0,
$$

$i=1, \ldots, n$, where $u:=\left(u_{1}, \ldots, u_{n}\right)$ and

$$
C_{i}\left(u_{i}\right)=\frac{\partial}{\partial u_{i}}\left\{\frac{3}{2} k_{B} N_{i}\left(u_{i}\right) u_{i}+\delta_{i 1} \widetilde{E}\left(u_{i}\right)\right\},
$$

with $\delta_{i 1}$ being the Kronecker's delta.

ASSUMPTION 3. Assume that the function $F_{1}\left(u_{1}\right)$ has exactly three zeros: 0,1 and $U_{0} \in(0,1)$ such that $F_{1}^{\prime}(0)<0, F_{1}^{\prime}\left(U_{0}\right)>0$ and $F_{1}^{\prime}(1)<0$.

ASSUMPTION 4. $\sup _{i \in\{2, \ldots, n\}} \sup _{u \in[-1,2]^{n}}\left(\left|K_{i}(u)\right|+\left|D K_{i}(u)\right|\right)<\tau$ with $\tau$ sufficiently small, $K_{i}(\mathbf{0})=0$ for all $i \in\{2, \ldots, n\}$.

This assumption is reasonable, as both the absorption of energy (in the process of so called Inverse BremsStrahlung) and the energetic losses are almost entirely carried out in the electron component.

ASSUMPTION 5. $c_{i j}(u)>0, c_{i j}(u)=c_{j i}(u)$ for all $i, j \in\{1, \ldots, n\}, u \in \mathbb{R}^{n}$. For all $i, k \in\{1, \ldots, n\}, k \neq i$ and al $u_{i}, u_{j} \in[0,1]$, we have $\sum_{j \neq i} c_{i j, k}(u)\left(u_{j}-u_{i}\right)+c_{i k}(u)>0$.

This assumption may be justified by the fact that the derivatives $c_{i j, k}(u)$ are relatively large only for small values of $u$ thus they are, in a way, damped by the factors $\left(u_{i}-u_{j}\right)$.

ASSUMPTION 6. $C_{i}\left(u_{i}\right)>C_{0 i}>0$ for all $u_{i} \in[0,1]^{n}$.

ASSUMPTION 7. For all $u \in[-1,2]^{n}$ and $i \in\{1, \ldots, n\},\{\vec{v}(u) \cdot \vec{n}\}_{i, u_{j}} \leq 0$ for all $j \neq i$.

This is a simplifying technical condition. It can be fulfilled e.g., if we assume that $\vec{v}_{i}(u)=\vec{v}_{i}\left(u_{i}\right)$. In view of Assumption 6 system (7.4) satisfies condition (2.1) of Section 2. It also satisfies point 4. of Assumption 1.

Now, we will show that Assumptions 3, 4, 5 imply Assumption 2. We have for $i \neq 1, k \neq i$,

$$
f_{i, k}(u)=\sum_{j \neq i} c_{i j, k}\left(u_{j}-u_{i}\right)+c_{i k}(u)+K_{i, k}(u),
$$

whereas for $i=1, k \neq 1$

$$
f_{1, k}(u)=\sum_{j \neq i} c_{1 j, k}\left(u_{j}-u_{i}\right)+c_{1 k}(u)
$$

From Assumption 5 it follows that for $\tau>0$ sufficiently small $f_{i, k}(u)>0$. Thus the monotonicity condition is satisfied. Also the other conditions of Assumption 2 are satisfied. To prove this we must examine the roots of the function $f(u)$ and the structure of eigenvalues of $D f$ at these roots. First, 
using the fact that the terms $K_{i}(u)$ are assumed sufficiently small, we will analyze the solutions to the simplified system of the form:

$$
\begin{array}{r}
F_{1}\left(u_{1}\right)+\sum_{j \neq 1} c_{1 j}(u)\left(u_{j}-u_{1}\right)=0 \\
\sum_{j \neq i} c_{i j}(u)\left(u_{j}-u_{i}\right)=0,
\end{array}
$$

where $i=2, \ldots, n$.

LEMMA 11. The only solutions to system (7.5) are $(0, \ldots, 0),(1, \ldots, 1)$ and $\left(U_{0}, \ldots, U_{0}\right)$.

PROOF. Adding the equations and using the symmetry $c_{i j}=c_{j i}$, we obtain:

$$
F_{1}\left(u_{1}\right)=0 .
$$

Hence the first component of the solution to system (7.5) is equal to one of the solutions to Eq.(7.6). The set of $n-1$ equations for $i=2, \ldots, n$ can be written in the form:

$$
\mathcal{N}_{n-1}\left(u_{2}, \ldots, u_{n}\right)^{T}=-u_{1}\left(c_{21}(u), \ldots, c_{n 1}(u)\right)^{T}
$$

where

$$
\mathcal{N}_{n-1}=\left(\begin{array}{cccc}
-\sum_{j \neq 2} c_{2 j}(u) & c_{23}(u) & \ldots & c_{2 n}(u) \\
c_{32}(u) & -\sum_{j \neq 3} c_{3 j}(u) & \ldots & c_{3 n}(u) \\
\ldots & \ldots & \ldots & \ldots \\
c_{n 2}(u) & c_{n 3}(u) & \ldots & -\sum_{j \neq n} c_{n j}(u)
\end{array}\right)
$$

Consider an auxiliary matrix arising from $\mathcal{N}_{n-1}$ by rejecting from the diagonal sums the terms $c_{i 1}$, i.e.

$$
\left(\begin{array}{cccc}
-\sum_{j \neq 1,2} c_{2 j}(u) & c_{23}(u) & \ldots & c_{2 n}(u) \\
c_{32}(u) & -\sum_{j \neq 1,3} c_{3 j}(u) & \ldots & c_{3 n}(u) \\
\ldots & \ldots & \ldots & \ldots \\
c_{n 2}(u) & c_{n 3}(u) & \ldots & -\sum_{j \neq 1, n} c_{n j}(u)
\end{array}\right) .
$$

The principal eigenvalue of this matrix is equal to 0 , whereas the eigenvector corresponding to this eigenvalue is equal to $(1, \ldots, 1)$. By using Lemma 3 in [8] we infer that all the eigenvalues of $\mathcal{N}_{n-1}$ will be negative, hence $\operatorname{det} \mathcal{N}_{n-1} \neq 0$. Thus system (7.7), for a given $u_{1}$ has exactly one solution. It is equal to $\left(u_{1}, \ldots, u_{1}\right)$, where $u_{1}$ satisfies the equation $F_{1}(y)=0$. The lemma is proved.

Now, let us find the structure of eigenvalues of $D f(\widetilde{u})$ for $\tau=0$ and $\widetilde{u}$ equal to $(0, \ldots, 0),(1, \ldots, 1)$ and $\left(U_{0}, \ldots, U_{0}\right) . D f(\widetilde{u})$ has the form:

$$
\operatorname{Df}(\widetilde{u})=\left(\begin{array}{cccc}
F_{1}^{\prime}\left(\widetilde{u}_{1}\right)-\sum_{j \neq 1} c_{1 j}(\widetilde{u}) & c_{12}(\widetilde{u}) & \ldots & c_{1 n}(\widetilde{u}) \\
c_{21}(\widetilde{u}) & -\sum_{j \neq 2} c_{2 j}(\widetilde{u}) & \ldots & c_{2 n}(\widetilde{u}) \\
\ldots & \ldots & \ldots & \ldots \ldots \\
c_{n 1}(\widetilde{u}) & c_{n 2}(\widetilde{u}) & \ldots & -\sum_{j \neq 1, n} c_{n j}(\widetilde{u})
\end{array}\right) .
$$

(Note that the terms proportional to $c_{i, k}(u)\left(\widetilde{u}_{i}-\widetilde{u}_{j}\right)$ vanish.) Let us consider the matrix: 


$$
\left(\begin{array}{cccc}
-\sum_{j \neq 1} c_{1 j}(\widetilde{u}) & c_{12}(\widetilde{u}) & \ldots & c_{1 n}(\widetilde{u}) \\
c_{21}(\widetilde{u}) & -\sum_{j \neq 2} c_{2 j}(\widetilde{u}) & \ldots & c_{2 n}(\widetilde{u}) \\
\ldots & \ldots & \ldots & \ldots \ldots \\
c_{n 1}(\widetilde{u}) & c_{n 2}(\widetilde{u}) & \ldots & -\sum_{j \neq 1, n} c_{n j}(\widetilde{u})
\end{array}\right)
$$

As before one notes that the principal eigenvalue of this matrix is equal to 0 , whereas the eigenvector corresponding to this eigenvalue is equal to $(1, \ldots, 1)$. Thus by means of Lemma 3 in [8] we have proved the following lemma.

LEMMA 12. For $\tau=0$ all the eigenvalues of $D f(\widetilde{u})$ have their real parts smaller than zero, if $F_{1}^{\prime}\left(\widetilde{u}_{1}\right)<0$ and larger than zero, if $F_{1}^{\prime}\left(\widetilde{u}_{1}\right)>0$.

Lemma 11 and the implicit function theorem imply the following lemma.

LEMMA 13. Assume that the function $F_{1}\left(u_{1}\right)$ has exactly three zeros: $\mathbf{0}, \mathbf{1}$ and $u_{0} \in(0,1)$. Then for all $\tau>0$ sufficiently small the only solutions to systems $f(u)$ with $f$ given by equations (7.2) are $(0, \ldots, 0),\left(\bar{u}_{1}, \ldots, \bar{u}_{n}\right)=(1, \ldots, 1)+O(\tau)$ and $\left(\hat{u}_{1}, \ldots, \hat{u}_{1}\right)=\left(u_{0}, \ldots, u_{0}\right)+O(\tau)$.

By means of this lemma and the fact that the eigenvalues of a matrix depend continuously on parameters we may prove the lemma corresponding to Lemma 12.

LEMMA 14. For $\tau$ sufficiently small all the eigenvalues of $D f(\widetilde{u})$, for $\widetilde{u}$ equal to one of the solutions of the equation $f(u)=0$, have their real parts smaller than zero, if $F_{1}^{\prime}\left(\widetilde{u}_{1}\right)<0$ and larger than zero, if $F_{1}^{\prime}\left(\widetilde{u}_{1}\right)>0$.

By the linear change of variables $u_{i} \rightarrow\left(\bar{u}_{i}\right)^{-1} u_{i}$ the largest root of system (7.2) becomes equal to $(1, \ldots, 1)$ and the intermediate one changes to $\left(u_{01}, \ldots, u_{0 n}\right)$.

We will construct a homotopy satisfying Assumption 2. We will divide this homotopy into three stages.

1. $\lambda \in\left[\frac{2}{3}, 1\right]:$ Let $\widetilde{c}_{i j}=\min _{u \in[-1,2]} c_{i j}(u), i, j \in\{1, \ldots, n\}$ and let

$$
c_{i j}(u)=\widetilde{c}_{i j}+c_{i j}^{*}(u) .
$$

Let

$$
f_{i}=F_{i}(u)+3\left(\lambda-\frac{2}{3}\right) K_{\lambda i}(u)+\sum_{j \neq i} h_{\lambda i j}(u)\left(u_{j}-u_{i}\right),
$$

where $F_{i}(u) \equiv 0$ for $i \in\{2, \ldots, n\}$ and

$$
h_{\lambda i j}(u)=\widetilde{c}_{i j}+3\left(\lambda-\frac{2}{3}\right) c_{i j}^{*}(u) .
$$

2. $\lambda \in\left[\frac{1}{3}, \frac{2}{3}\right]:$ 


$$
f_{i}=\sum_{j \neq i}\left[3\left(\frac{2}{3}-\lambda\right) H+3\left(\lambda-\frac{1}{3}\right) \widetilde{c}_{i j}\right]\left(u_{j}-u_{i}\right)
$$

where $H>0$ is sufficiently large.

3. $\lambda \in\left[0, \frac{1}{3}\right]:$

$$
f_{i}=\sum_{j \neq i} H\left(u_{j}-u_{i}\right)+3\left[\left(\frac{1}{3}-\lambda\right)+\lambda \delta_{i 1}\right] F_{1}\left(u_{i}\right) .
$$

It is obvious that for $\lambda \in\left[\frac{1}{3}, 1\right]$ Assumption 2 is satisfied. We will show that it is satisfied for $\lambda \in\left[0, \frac{1}{3}\right]$. It is sufficient to verify that the principal eigenvalue of the matrix $D f$ at $u_{ \pm}$is negative. As before we can replace the equation $f(u)=0$ by

$$
\begin{gathered}
F_{1}\left(u_{1}\right)+3\left(\frac{1}{3}-\lambda\right)\left(F_{1}\left(u_{2}\right)+\ldots+F_{1}\left(u_{n}\right)\right)=0 \\
\mathcal{N}_{n-1}^{*}\left(u_{2}, \ldots, u_{n}\right)^{T}=-u_{1}(1, \ldots, 1)^{T}-H^{-1} 3\left(\frac{1}{3}-\lambda\right)\left(F_{1}\left(u_{2}\right), \ldots, F_{1}\left(u_{n}\right)\right)^{T},
\end{gathered}
$$

where $\mathcal{N}_{n-1}^{*}$ is an $(n-1) \times(n-1)$ matrix:

$$
\mathcal{N}_{n-1}^{*}=\left(\begin{array}{cccc}
-(n-1) & 1 & \ldots & 1 \\
1 & -(n-1) & \ldots & 1 \\
\ldots & \ldots & \ldots & \ldots \\
1 & 1 & \ldots & -(n-1)
\end{array}\right)
$$

Let us note that $\left|\operatorname{det} \mathcal{N}_{n-1}^{*}\right|=n^{n-2}$. Hence due to the implicit function theorem for $H>0$ sufficiently large and given the right hand sides there exists a unique solution $\left(u_{2}, \ldots, u_{n}\right)$ of system (7.12). This solution is equal to $\left(u_{1}, \ldots, u_{1}\right)+3\left(\frac{1}{3}-\lambda\right) O\left(H^{-1}\right)$. Putting this relation into (7.11) we obtain $F_{1}\left(u_{1}\right)+\left(\frac{1}{3}-\lambda\right) \sum_{i \neq 1} F_{1}\left(u_{i}\right)=F_{1}\left(u_{1}\right)\left(1+(n-1)\left(\frac{1}{3}-\lambda\right)\right)+(n-1)\left(\frac{1}{3}-\lambda\right) O\left(H^{-1}\right)=$ $(n-1)\left(\frac{1}{3}-\lambda\right) O\left(H^{-1}\right)$. By the use of the implicit function theorem we conclude that for every solution $\left(u_{1}, \ldots, u_{n}\right)$ to $(7.11) u_{1}$ is equal to one of the states $\mathbf{0}, u_{0}, \mathbf{1}$, plus $O\left(H^{-1}\right)$ terms. Hence in system $(7.12)\left(F_{1}\left(u_{2}\right), \ldots, F_{1}\left(u_{n}\right)\right)^{T}=(0, \ldots, 0)^{T}+O\left(H^{-1}\right)$. This implies that $u_{i}=u_{1}+O\left(H^{-2}\right)$, $i \in\{2, \ldots, n\}$. Now, we may succesively repeat the procedure, to conclude that $u_{i}=u_{1}+O\left(H^{-k}\right)$ for any natural $k$. This implies that $u_{i}=u_{1}, i \in\{2, \ldots, n\}$ for all $\lambda \in\left[0, \frac{1}{3}\right]$. Thus Assumption 2 is satisfied for all $\lambda \in[0,1]$.

Consequently using Theorem 1 we can state the following result.

THEOREM 4. Suppose that all the functions in system (7.4) are sufficiently smooth and that Assumptions 3 - 7 are fulfilled. Then there exists $q^{*} \in \mathbb{R}^{1}$ such that for $q=q^{*}$ system (7.4) has a strictly monotone unique (up to translation in $\xi$ ) heteroclinic solution joining the states $\mathbf{0}$ and $\mathbf{1}$. 


\section{Equations with delay}

In this section we study existence of solutions of the problem (1.3), (1.4) with a small perturbation by terms with delay. Consider the system

$$
a_{i}\left(u_{i}, u_{i}^{\prime}\right) u_{i}^{\prime \prime}-q c_{i}\left(u_{i}, u_{i}^{\prime}\right) u_{i}^{\prime}+M_{i}\left(u, u_{i}^{\prime}\right) u_{i}^{\prime}+f_{i}(u)+P_{i}(\tau, q, u)=0,
$$

$i=1, \ldots, n$, where the operator $P_{i}: \mathbb{R}^{l} \times \mathbb{R}^{1} \times B_{20} \rightarrow B_{0}, l \geq 1$ and $P_{i}(0, q, u) \equiv 0$. Let us assume that for $\tau=0$ there exists a strictly monotone heteroclinic pair $(S, U)$ joining the states $\mathbf{0}$ and $\mathbf{1}$ satisfying system (8.1) and that $P$ is continuously Fréchet differentiable with respect to $(q, u)$ in some open neighbourhood of the solution triple $(0, S, U)$.

Using the results of Sections 3, 4 we can state the following theorem of existence.

THEOREM 5. Let conditions of Section 2 be satisfied. Then for all $\tau$ with $|\tau|$ sufficiently small there exists a solution pair $\left(q_{\tau}, u_{\tau}\right) \in \mathbb{R}^{1} \times B_{20}$ satisfying system (8.1) such that

$$
\left|q_{\tau}-S\right|+\left\|u_{\tau}-U\right\|_{B_{20}} \rightarrow 0 \quad \text { as } \quad|\tau| \rightarrow 0 .
$$

We do not use in this theorem all conditions of Section 2. It remains valid if we assume that the monotonicity conditions (1.5) are satisfied and that there exists a solution for $\tau=0$.

As an example, let us consider a system of equations with delays:

$$
\begin{gathered}
G(\tau, q, u):=a_{i}\left(u_{i}(\xi), u_{i}^{\prime}(\xi)\right) u_{i}^{\prime \prime}(\xi)-q c_{i}\left(u_{i}(\xi), u_{i}^{\prime}(\xi)\right) u_{i}^{\prime}(\xi)+ \\
M_{i}\left(u(\xi), u_{i}^{\prime}(\xi)\right) u_{i}^{\prime}(\xi)+f_{i}\left(u_{1}\left(\xi-\phi_{i 1}(q) \tau\right), \ldots, u_{n}\left(\xi-\phi_{i n}(q) \tau\right)\right)=0,
\end{gathered}
$$

$i=1, \ldots, n$, where $\phi_{i j}$ are smooth functions of $q$ and $\tau \in \mathbb{R}^{1}$. We may rewrite the system as

$$
\begin{gathered}
a_{i}\left(u_{i}^{\prime}(\xi)\right) u_{i}^{\prime \prime}(\xi)-q c_{i}\left(u_{i}(\xi), u_{i}^{\prime}(\xi)\right) u_{i}^{\prime}(\xi)+M_{i}\left(u(\xi), u_{i}^{\prime}(\xi)\right) u_{i}^{\prime}(\xi)+ \\
f_{i}(u(\xi))+P_{i}(\tau, q, u(\xi))=0
\end{gathered}
$$

where

$$
P_{i}(\tau, q, u(\xi))=f_{i}\left(u_{1}\left(\xi-\phi_{i 1}(q) \tau\right), \ldots, u_{n}\left(\xi-\phi_{i n} \tau\right)\right)-f_{i}(u(\xi)) .
$$

Let us note that $G:=\left(G_{1}, \ldots, G_{n}\right)$ acts from the Banach space $\mathbb{R}^{1} \times \mathbb{R}^{1} \times B_{20}$ to the space $B_{0}$. Moreover, it is Fréchet differentiable. The $i$-th component of the Fréchet derivative of the perturbation term $P$ at the point $\left(\tau_{0}, q_{0}, u_{0}\right)$ is equal to the following operator:

$$
\begin{gathered}
(D P)_{i}\left(\tau_{0}, q_{0}, u_{0}\right)(\delta \tau, \delta q, \delta u(\xi))=-\sum_{j=1}^{n} f_{i, j}\left(u_{0}(\xi)\right) \delta u_{j}(\xi)+ \\
\sum_{j=1}^{n} f_{i, j}\left(u_{01}\left(\xi-\phi_{i 1}\left(q_{0}\right) \tau_{0}\right), \ldots, u_{0 n}\left(\xi-\phi_{i n}\left(q_{0}\right) \tau_{0}\right)\right) \delta u_{j}\left(\xi-\phi_{i j}\left(q_{0}\right) \tau_{0}\right)- \\
\sum_{j=1}^{n} f_{i, j}\left(u_{01}\left(\xi-\phi_{i 1}\left(q_{0}\right) \tau_{0}\right), \ldots, u_{0 n}\left(\xi-\phi_{i n}\left(q_{0}\right) \tau_{0}\right)\right) \phi_{i j}^{\prime}\left(q_{0}\right) u_{0 j}^{\prime}\left(\xi-\phi_{i j}\left(q_{0}\right) \tau_{0}\right) \tau_{0} \delta q- \\
\sum_{j=1}^{n} f_{i, j}\left(u_{01}\left(\xi-\phi_{i 1}\left(q_{0}\right) \tau_{0}\right), \ldots, u_{0 n}\left(\xi-\phi_{i n}\left(q_{0}\right) \tau_{0}\right)\right) \phi_{i j}\left(q_{0}\right) u_{0 j}^{\prime}\left(\xi-\phi_{i j}\left(q_{0}\right) \tau_{0}\right) \delta \tau .
\end{gathered}
$$


To prove it let us note that we have

$$
\begin{gathered}
\left|P\left(\tau_{0}+\delta \tau, q_{0}+\delta q, u_{0}+\delta u\right)-D P\left(\tau_{0}, q_{0}, u_{0}\right)(\delta \tau, \delta q, \delta u(\xi))\right|=o(|\delta u(\xi)|)+ \\
o\left(\sum_{i j=1}^{n}\left|\delta u_{i}\left(\xi-\phi_{i j}\left(q_{0}\right) \tau_{0}\right)\right|\right)+o(|\delta q|)+o(|\delta \tau|) .
\end{gathered}
$$

Now, $\sup _{\xi \in \mathbb{R}^{1}}\left|\delta u_{i}\left(\xi-\phi_{i j}\left(q_{0}\right) \tau_{0}\right)\right|=\sup _{\xi \in \mathbb{R}^{1}}\left|\delta u_{i}(\xi)\right|$, so the right hand side of the last equality behaves like $o\left(|\delta \tau|+\|\delta u(\xi)\|_{C^{0}\left(\mathbb{R}^{1}\right.}+|\delta q|\right)$.

The same considerations are valid, when $P_{i}$ are of the form:

$$
\begin{gathered}
P_{i}(\tau, q, u(\xi))=-q \widetilde{c}_{i}\left(u_{i}(\xi), u_{i}^{\prime}(\xi), u_{i}\left(\xi-\chi_{i}(q) \tau\right), u_{i}^{\prime}\left(\xi-\chi_{i}(q) \tau\right)\right) u_{i}^{\prime}\left(\xi-\mu_{i}(q) \tau\right)+ \\
q c_{i}\left(u_{i}(\xi), u_{i}^{\prime}(\xi)\right) u_{i}^{\prime}(\xi)+\widetilde{M}_{i}\left(u_{1}(\xi), \ldots, u_{n}(\xi), u_{1}\left(\xi-\kappa_{i 1}(q) \tau\right), \ldots,\right. \\
\left.u_{n}(\xi), u_{n}\left(\xi-\kappa_{i n}(q) \tau\right), u_{i}^{\prime}(\xi), u_{i}^{\prime}\left(\xi-k_{i}(q) \tau\right)\right) u_{i}^{\prime}\left(\xi-k_{i}^{*}(q) \tau\right)- \\
M_{i}\left(u(\xi), u_{i}^{\prime}(\xi)\right) u_{i}^{\prime}(\xi)+\widetilde{f}_{i}\left(u_{1}(\xi), \ldots, u_{n}(\xi), u_{1}\left(\xi-\phi_{i 1}(q) \tau\right), \ldots, u_{n}\left(\xi-\phi_{i n} \tau\right)\right)-f_{i}(u(\xi)),
\end{gathered}
$$

where

$$
\begin{gathered}
\widetilde{f}_{i}\left(u_{1}(\xi), \ldots, u_{n}(\xi), u_{1}(\xi), \ldots, u_{n}(\xi)\right) \equiv f_{i}(u(\xi)), \\
\widetilde{c}_{i}\left(u_{i}(\xi), u_{i}^{\prime}(\xi), u_{i}(\xi), u_{i}^{\prime}(\xi)\right) \equiv c_{i}\left(u_{i}(\xi), u_{i}^{\prime}(\xi)\right)
\end{gathered}
$$

and

$$
\widetilde{M}_{i}\left(u_{1}(\xi), \ldots, u_{n}(\xi), u_{1}(\xi), \ldots, u_{n}(\xi), u_{i}^{\prime}(\xi), u_{i}^{\prime}(\xi)\right) \equiv M_{i}\left(u(\xi), u_{i}^{\prime}(\xi)\right)
$$

Acknowledgments. BK was partially supported by NSF grant IBN-0083653 under the Biocomplexity Initiative and by the Interdisciplinary Center for the Study of Biocomplexity. The authors thank the Polish-French programme Polonium for the opportunity of mutual visits.

\section{References}

[1] C.C. CONLEY, R. GARDNER, An application of the generalized Morse index to travelling wave solutions of a competitive reaction diffusion model, Indiana Univ. Math. J., 33 (1989), 319-343.

[2] W.A. COPPEL, Dichotomies in stability theory, Lecture Notes in Math. 629, Springer, 1978.

[3] M. CRANDALL An Introduction to constructive aspects of bifurcation theory and implicit function theorem, (in) Applications of bifurcation theory, ed. P.Rabinowitz, Acad.Press,New York, 1977.

[4] E.C.M. CROOKS, J.F. TOLAND, Travelling waves for reaction diffusion convection systems, Top. Meth. in Nonl. Anal., 11 (1998), 19-43.

[5] E.C.M. CROOKS, On the Volperts' theory of travelling wave solutions for parabolic equations, Nonl. Anal., TM\&A, 26 (1996), 1621-1642. 
[6] W. ECKHAUS, A. VAN HARTEN, Z. PERADZYŃSKI, A Singularly perturbed free boundary problem describing a laser sustained plasma, SIAM J. Appl. Math. 45 (1985), 1-31.

[7] W. ECKHAUS, A. VAN HARTEN, Z. PERADZYŃSKI, Plasma produced by a laser in a medium with convection and free surface satisfying a hamilton-jacobi equations, Physica $\mathbf{D}$, 27 (1987), 90-112.

[8] B. KAŹMIERCZAK, Existence of travelling wave solutions for reaction-diffusion convection systems via the Conley index theory, Top. Meth. in Nonl. Anal., 17(2) (2001), 359-403.

[9] B. KAŹMIERCZAK, Heteroclinic connections in a realistic model of laser sustained plasma, Nonlinear Analysis TM\&A, 29 (1997), 247-264.

[10] B. KAŹMIERCZAK, Z. PERADZYŃSKI, Heteroclinic solutions for a system of strongly coupled ODEs, Math.Meth.in Appl.Scien., 19 (1996), 451-461.

[11] K.MISCHAIKOV, V.HUTSON, Travelling waves for mutualist species, SIAM J. Math. Anal., 24 (1993), 987-1008.

[12] K.J.PALMER, Exponential dichotomies and transversal homoclinic points, J. Diff. Equat., 20 (1984), 225-256.

[13] Z. PERADZYŃSKI, Continuous optical discharge, properties and modeling, Invited papers, ICPIG XXI, Bochum , 1993.

[14] A.E. TAYLOR, Introduction to functional analysis, J.Wiley and Sons, New York, 1958.

[15] A.I. VOLPERT, V.A. VOLPERT, Applications of the rotation theory of vector fields to the study of wave solutions of parabolic equations. Trans. Moscow Math. Soc., 52 (1990), 59-108.

[16] A.,V., VOLPERT V., Travelling wave solutions of parabolic systems, AMS Providence, 1994.

[17] A.I. VOLPERT, V.A. VOLPERT, The construction of the Leray-Schauder degree for elliptic operators in unbounded domains. Annales de l'IHP. Analyse non lineaire, 11 (1994), 245-273.

[18] V. VOLPERT, A. VOLPERT, Location of spectrum and stability of solutions for monotone parabolic systems, Adv. Diff. Eq., 2 (1997), 811-830.

[19] V. VOLPERT, A. VOLPERT, J.F. COLLET, Topological degree for elliptic operators in unbounded cylinders. Adv. Diff. Eq., 4 (1999), 777-812.

[20] V. VOLPERT, A. VOLPERT, Existence and stability of multidimensional travelling waves in the monostable case. Israel Journal of Mathematics, 110 (1999), 269-292.

[21] A. VOLPERT, V. VOLPERT, Existence of multidimensional travelling waves and systems of waves. Comm. PDE, 26 (2001), 421-459. 